\title{
Optical Fiber Sensor Technologies for Efficient and Economical Oil Recovery
}

\section{Technical Progress Report}

Reporting Period Start Date: 1 October 2001

Reporting Period End Date: 30 September 2002

Principal Authors: Kristie Cooper, Gary Pickrell and Anbo Wang

Report Issued: April 2003

DOE Award Number: DE-FG26988C15167

Submitted by: Center for Photonics Technology

Bradley Department of Electrical Engineering

Virginia Polytechnic Institute \& State University

Blacksburg, VA 24061-0111

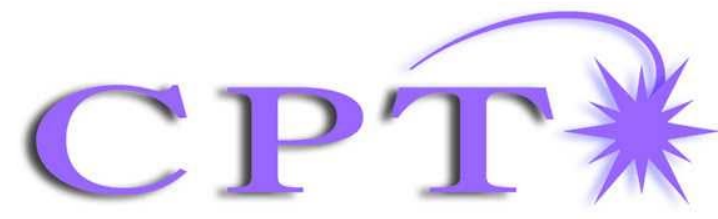

CENTER FOR PHOTONICS TECHNOLOGY 


\section{Disclaimer:}

This report was prepared as an account of work sponsored by an agency of the United States Government. Neither the United States Government nor any agency thereof, nor any of their employees, makes any warranty, express or implied, or assumes any legal liability or responsibility for the accuracy, completeness, or usefulness of any information, apparatus, product, or process disclosed, or represents that its use would not infringe privately owned rights. Reference herein to any specific commercial product, process, or service by trade name, trademark, manufacturer, or otherwise does not necessarily constitute or imply its endorsement, recommendation, or favoring by the United States Government or any agency thereof. The views and opinions of authors expressed herein do not necessarily state or reflect those of the United States Government or any agency thereof. 


\section{Executive Summary}

This report summarizes technical progress over the fourth year of the "Optical Fiber Sensor Technologies for Efficient and Economical Oil Recovery" program, funded by the Federal Energy Technology Center of the U.S. Department of Energy, and performed by the Center for Photonics Technology of the Bradley Department of Electrical and Computer Engineering at Virginia Tech.

During the reporting period, research efforts under the program were focused on the development and evaluation of the fiber optic flow sensor system, and field testing in Tulsa, $\mathrm{OK}$ and the second field test of the pressure and temperature sensors in Coalinga, CA. The feasibility of a self-compensating fiber optic flow sensor based on a cantilever beam and interferometer for real-time flow rate measurements in the fluid filled pipes of oil field was clearly demonstrated. In addition, field testing of the pressure and temperature sensors deployed downhole continued. These accomplishments are summarized here.

- Theoretical analysis and simulations were performed to ensure performance of the design.

- The sensor fabrication and packaging techniques were investigated and improved.

- Prototype flow sensors were fabricated based on the fabrication experience of hundreds of test sensors.

- A lab-scale flow testing system was constructed and used for sensor evaluation.

- Field-testing was performed in both the indoor and outdoor flow testing facility at the University of Tulsa, OK.

- Testing of a multimode white light pressure and temperature sensor system continued at the oil site of Chevron/TexacoCompany (Coalinga CA). 


\section{Table of Contents}

Executive Summary ................................................................................................. ii

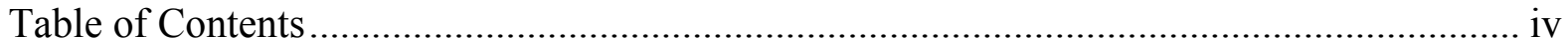

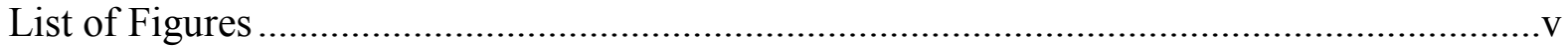

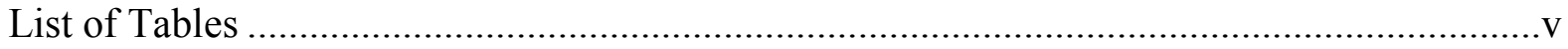

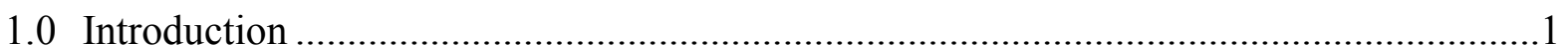

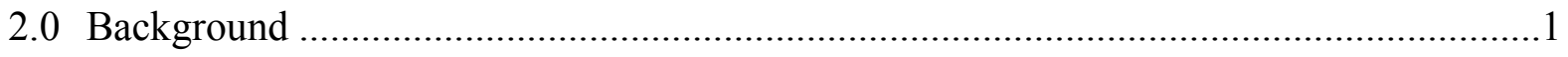

3.0 Self-Compensating Fiber Optic Flow Sensor System .............................................4

3.1 Operational Principle of Flow Sensor..................................................................... 4

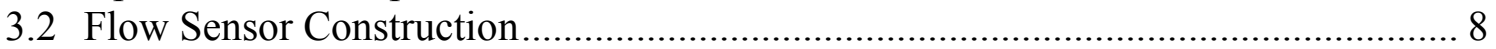

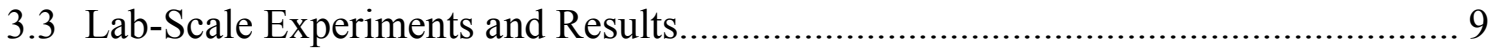

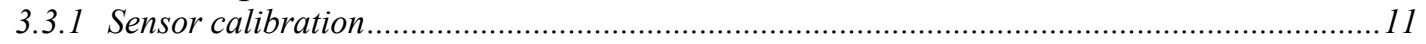

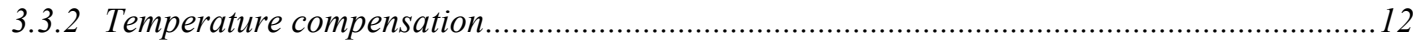

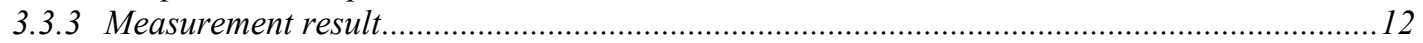

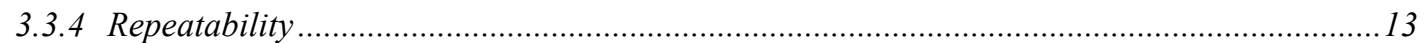

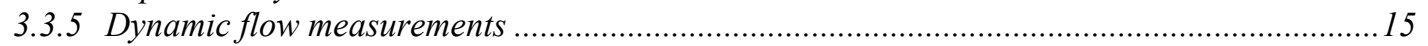

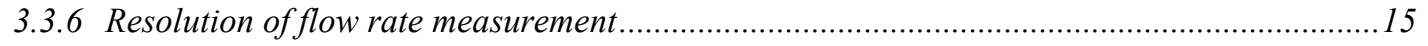

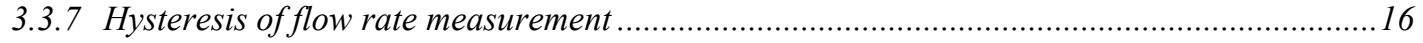

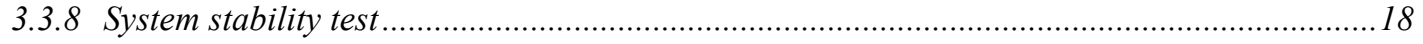

4.0 Field Testing of Flow Sensor System.................................................................19

4.1 Indoor Flow Testing......................................................................................... 19

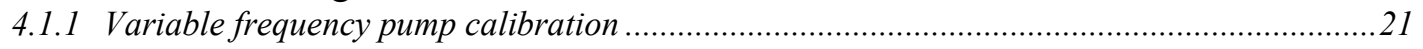

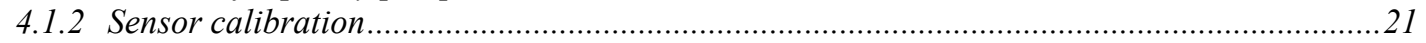

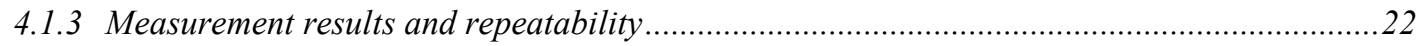

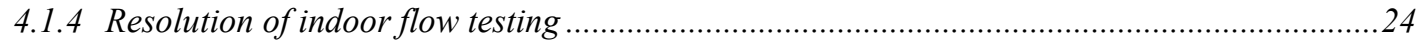

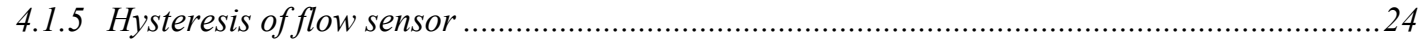

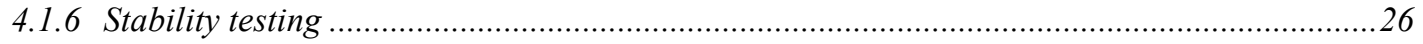

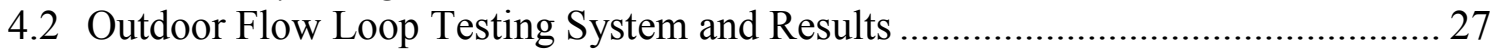

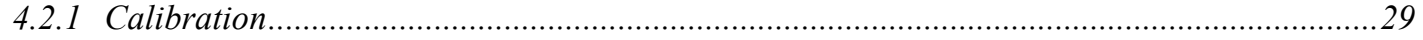

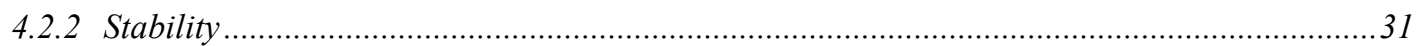

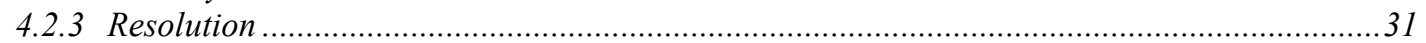

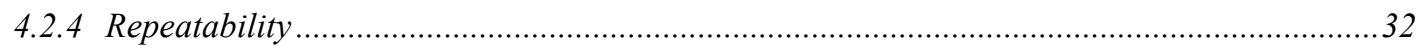

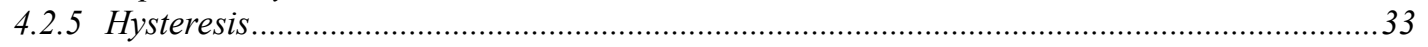

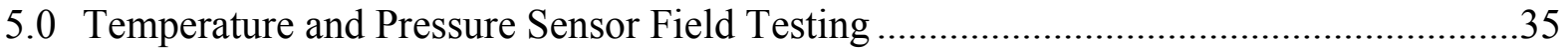

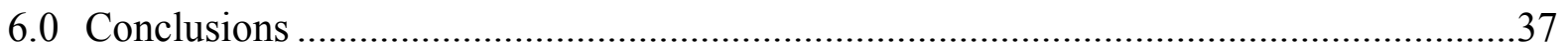

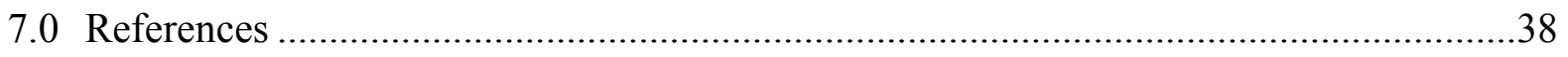

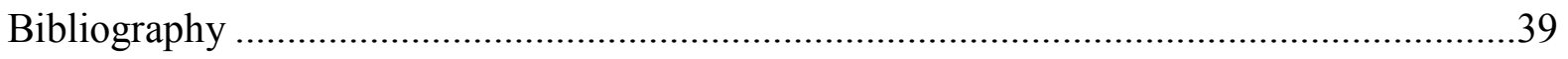

List of Acronyms and Abbreviations ..................................................................... 41 


\section{List of Figures}

Figure 2.1. Illustration of the principle of the self-calibrated interferometric/intensity-based

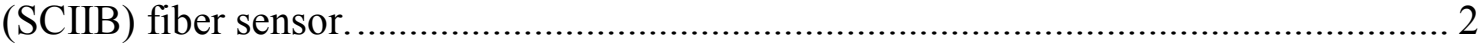

Figure 2.2. Illustration of the two channel outputs of the SCIIB sensor. ............................ 3

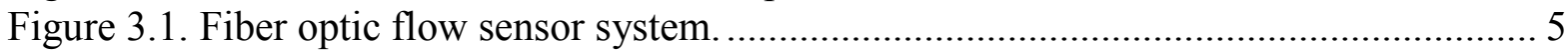

Figure 3.2. Simulation of flow sensor self-compensation. ............................................ 7

Figure 3.5. Fabrication of flow sensor........................................................................ 9

Figure 3.7. Lab-scale flow testing system setup....................................................... 10

Figure 3.9. Flow sensor testing setup in Center for Photonics Technology, Virginia Tech... 10

Figure 3.11. Relationship between flow rate and flow sensor air gaps. .............................. 11

Figure 3.13. Flow sensor calibration curve................................................................. 12

Figure 3.15. Fiber optic flow sensor self-compensation result...................................... 13

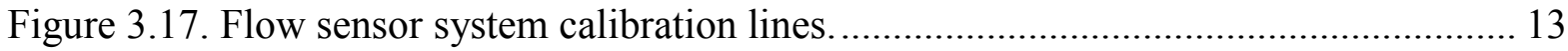

Figure 3.19. Flow measurement repeatability test results............................................... 14

Figure 3.21. Dynamic response of flow sensor............................................................. 15

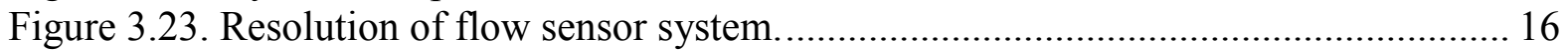

Figure 3.25. Hysteresis of flow sensor in lab-scale testing........................................... 17

Figure 3.27. Flow sensor system stability test over 12 hours at 7.2 GPM......................... 18

Figure 4.1. Indoor flow testing system in University of Tulsa, OK. .............................. 20

Figure 4.3. Variable frequency pump calibration curve using FT-24NENW flow meter. .... 21

Figure 4.5. Flow sensor calibration curve for indoor flow testing in Tulsa, OK.................. 22

Figure 4.7. Measurement results from fiber flow sensor in indoor testing loop.................. 23

Figure 4.9. Resolution of flow sensor system, indoor flow testing. ................................ 24

Figure 4.11. Flow sensor hysteresis measurement................................................... 25

Figure 4.13. Flow system stability test over approximately 2 hours. ............................... 26

Figure 4.15. Outdoor flow testing facility in Tulsa University, OK................................ 28

Figure 4.17. Fiber optic flow sensor deployment in outdoor flow loops of Tulsa University,

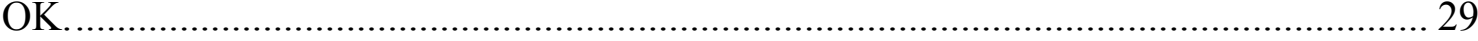

Figure 4.19. Fiber optic flow sensor control system for outdoor flow testing..................... 29

Figure 4.21. Flow sensor calibration for outdoor flow testing. ...................................... 30

Figure 4.23. Flow sensor stability during outdoor flow testing.................................... 31

Figure 4.25. Resolution of fiber flow sensor during outdoor flow testing. ......................... 32

Figure 4.27. Measurement results and repeatability testing in outdoor flow testing............. 33

Figure 4.28. Hysteresis testing in outdoor flow testing. ............................................. 34

Figure 5.1. Pressure sensor output from 6/3/2002 - 10/31/2002 ....................................... 35

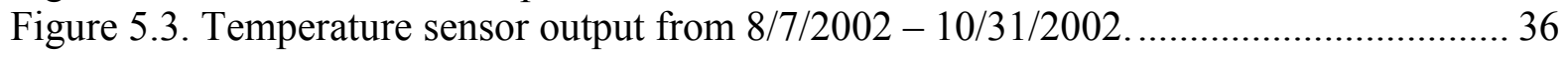

\section{List of Tables}

Table 3.1. Parameters of fiber optic flow sensor used for lab scale testing.......................... 11

Table 4.1. Parameters of fiber optic flow sensor used for indoor flow testing in Tulsa........ 19

Table 4.3. Fiber optic flow sensor parameters for outdoor flow testing in Tulsa, OK.......... 27

Table 6.1. Summary of the performance evaluation results for SCIIB flow sensor system... 37 


\subsection{Introduction}

This is the fourth annual report for the Virginia Tech Center for Photonics Technology (VTCPT) research project "Optical Fiber Sensor Technologies for Efficient and Economical Oil Recovery." The main objective for this four and one-half year program is the development and demonstration of cost-effective, reliable optical fiber sensors for the measurement of temperature, pressure, flow, and acoustic waves in downhole environments for use in oil recovery.

The sensors being developed under this program are based on the Self-Calibrated Interferometric/Intensity-Based (SCIIB) fiber optic sensor configuration invented at the Center for Photonics Technology. The SCIIB design measures displacement (elongation or contraction) of a small Fabry-Perot interferometer cavity constructed in an optical fiber. The Fabry-Perot assembly is typically cylindrical, and generally has dimensions on the order of $0.1 \mathrm{~mm}$ in diameter and $5 \mathrm{~mm}$ long. By proper mechanical design of the assembly, physical parameters such as pressure, temperature, and flow can be made to induce a displacement in the Fabry-Perot assembly, which may then be measured by the SCIIB system. In the SCIIB configuration, the Fabry-Perot assembly is designed so that the output of the cavity over the sensor's dynamic range is limited to the quasi-linear region of the interference signal output of the cavity. In this way, interpretation of the nonlinear output of typical interferometers is avoided, simplifying the signal processing.

The SCIIB system uses optical filtering to process the optical signal output so that two signals are produced: one which contains information about the cavity displacement, and another that contains information regarding undesired effects in the first signal, such as fluctuations in the optical source power, or changes in optical fiber loss. The second signal is used as a reference to eliminate these undesired effects, and provide self-calibration functions in the sensor.

During the reporting period, research efforts under the program were focused on the development and evaluation of the fiber optic flow sensor system, and field testing in Tulsa, OK and Coalinga, CA. Progress is described in detail in this report.

\subsection{Background}

The self-calibrated interferometric/intensity-based (SCIIB) fiber optic sensor successfully combines the advantages of both the interferometric and the intensity-based fiber sensors in a single system. Through a proper sensor design, the SCIIB technology can provide absolute measurement of various parameters with the full self-compensation capability for the source power fluctuation and the fiber loss changes. 
The basic SCIIB configuration is shown in Figure 2.1. The light from an optical source is launched into a two-by-two fiber coupler and propagates along the optical fiber to the sensor head. As shown in the inset of Figure 2.1, the input fiber and a reflector are thermally fused to a hollow glass tube with a predetermined air gap separation. By thermally fusing the glass capillary tube to the glass fibers, the use of organic adhesives such as epoxy is avoided, eliminating the temperature limits and temperature-dependent mechanical properties and hysteresis of those adhesives. The incident light is first partially reflected at the endface of the input fiber $\left(R_{1}\right.$ in Figure 2.1). The remainder of the light propagates across the air gap to the reflector surface where a second reflection $\left(R_{2}\right)$ is generated. The two reflections travel back along the same input fiber through the same coupler to the photodetection end.

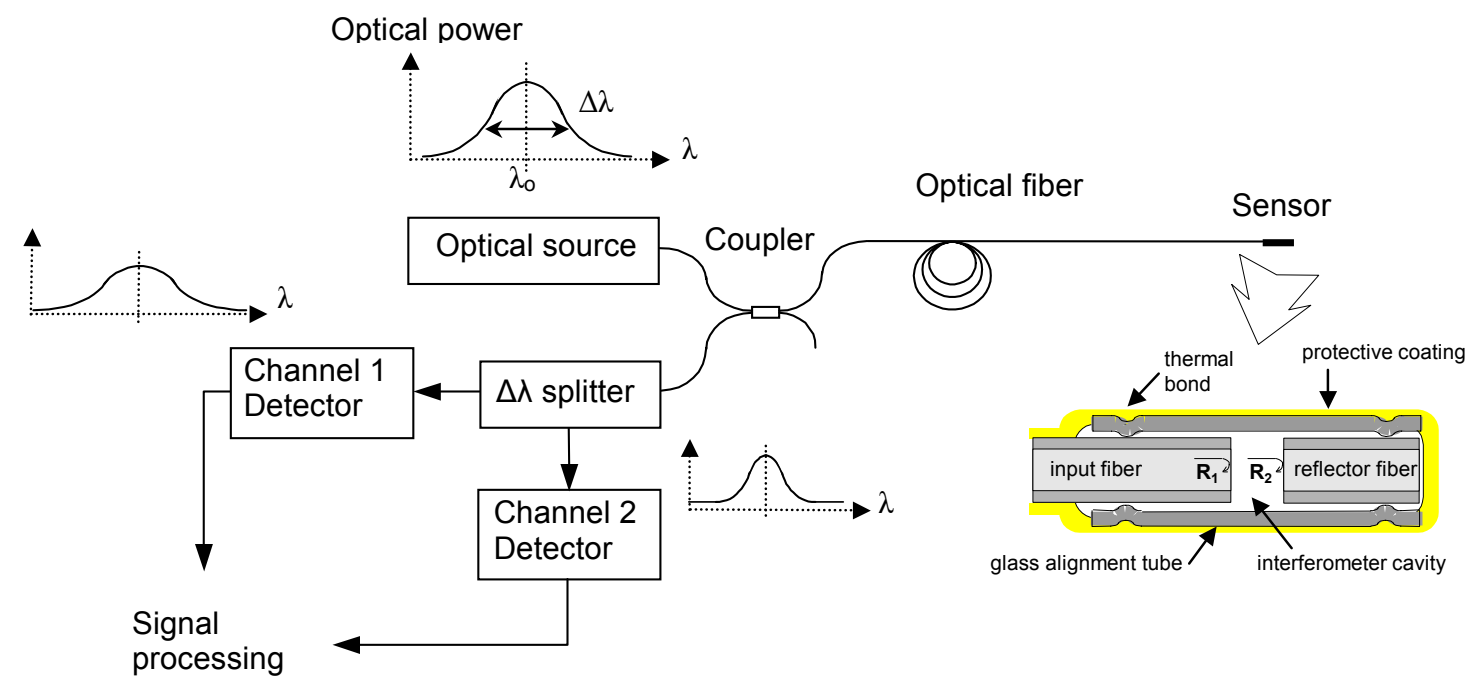

Figure 2.1. Illustration of the principle of the self-calibrated interferometric/intensity-based (SCIIB) fiber sensor.

In order to overcome problems associated with conventional fiber interferometric sensors, such as relative measurement, low frequency response, and complexity in signal demodulation, a special optoelectronic signal demodulation method is employed that allows self-calibrating absolute measurement with high frequency response. When the source coherence length is longer than the path length difference of the two legs in an interferometer, interference is observed when the two reflections are combined; when it is less than the path length difference, interference does not occur, and the combination of the two beams results in the sum of their intensities. In the SCIIB system, the coherence length of the sensor channel (Channel 2 in Figure 2.1) is engineered to exceed the path length difference in the Fabry-Perot cavity (twice the gap length), resulting in interference fringes as the gap length is changed by the measurand of interest. The coherence length of the reference channel (Channel 1 in Figure 2.1) is adjusted to be much shorter than the path length difference in the Fabry-Perot cavity. Therefore, no interference occurs and the power output of the reference channel is simply the sum of the optical power of the two reflections $\mathrm{R}_{1}$ and $\mathrm{R}_{2}$. Both channels are affected by changes in fiber loss, bending, connector loss and 
fluctuations in source power, while only the sensor channel exhibits changes due to the effect of the desired measurand, e.g. temperature or pressure, on the gap length. As shown in Figure 2.2, when the cavity length changes, one photodetector exhibits an interference signal output while the other is relatively unchanged because the initial air-gap length is larger than the coherence length of the original source. The undesired fluctuations can be canceled out by taking the ratio of the two channels. To simplify the processing and interpretation of the sensor output, the sensor probe is designed so that the optical intensity of the output over the expected full range of the measurand remains within the quasi-linear portion of the interferometric output. This is accomplished by controlling the length, diameter, wall thickness, and the modulus of elasticity of the capillary alignment tube during sensor fabrication. In this way complicated signal processing involving fringe counting or spectral decomposition can be avoided, and a simple photodetector used.
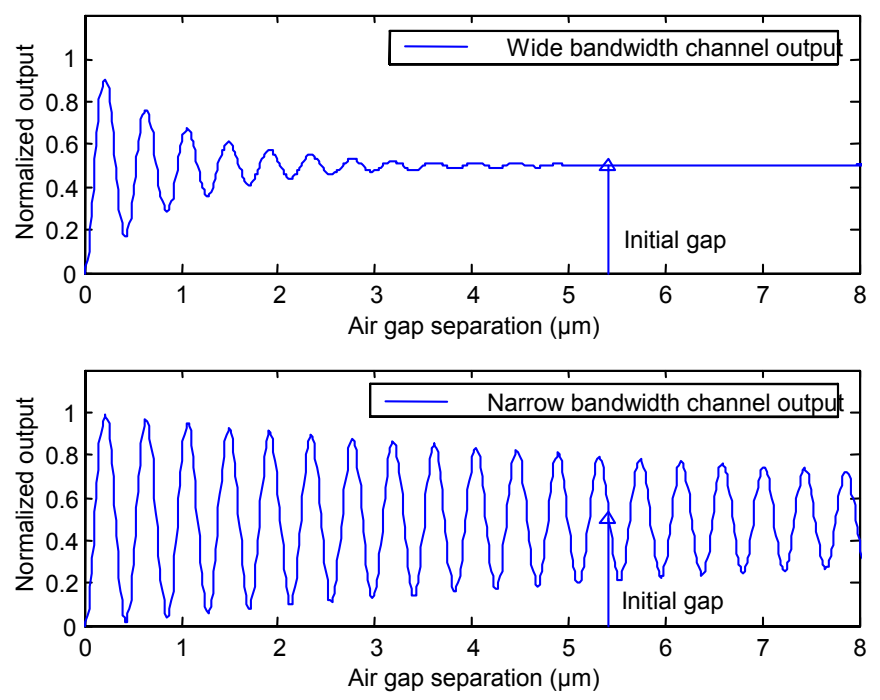

Figure 2.2. Illustration of the two channel outputs of the SCIIB sensor.

The SCIIB fiber optic sensor system can be used to measure different physical parameters, for instance, temperature, pressure, strain and acoustic waves. The sensor head can be specially designed to be only sensitive to the parameter of interests. In general, the measurand interacts with the sensor head and causes a change of the air gap. The SCIIB sensor system relates the air gap change to the measurand according to the physical principles governing the interaction, and directly gives the measuring results. 


\subsection{Self-Compensating Fiber Optic Flow Sensor System}

Flow rate is one of the key measurement parameters for both the downhole oil and surface piping systems of the oil production industry. Real-time, on-line monitoring of single-phase or multi-phase fluid flow rates with large dynamic range, high accuracy, good reliability and cost effectiveness is very important to realize the full economic potentiality of petroleum industries. Usually, conventional single-phase fluid flow meters with electric signal outputs are operated by combining electrical sensing elements with mechanical devices. The fluid flow is monitored by detecting some change in a particular parameter such as frequency of turbine, frequency of vortex shedding-induced vibration, flow induced pressure change, flow induced temperature change, etc. However, due to the harsh environment in the oil field including high temperature, high pressure, corrosive agents, electrical flow meters such as paddlewheel, turbine, vibration-based or Pito-tube flow meters are not suitable for oil field applications.

Based on the investigation of various flow meter configurations, and considering the especially harsh conditions of the downhole oil environment and the unique requirements of this application such as temperature insensitivity, pressure isolation, high flow rate sensitivity, and high dynamic range, a self-compensating fiber optic interferometric flow sensor system has been designed and developed. It features a unique combination of interferometric signal demodulation resulting in high resolution, self-compensation for temperature and pressure sensitivity, compactness, and simplified deployment (can be easily deployed through sidewall of the flow pipe) with very little disturbance. A prototype fiber optic flow sensor system was developed, and tested in the oil flow-testing center at Tulsa University. The laboratory and field-testing results demonstrate that this fiber optic sensor system can detect the flow rate of fluids with high resolution, reliability and stability.

\subsection{Operational Principle of Flow Sensor}

The basic principle of the self-compensating fiber optic flow sensor is illustrated in Figure 3.1. The system is composed of a sensor probe, optical spectrum analyzer (OSA) for optoelectronic signal processing and a dual core multimode fiber cable linking the sensor probe and the signal-processing unit. The broadband light from two light-emitting diodes (LEDs) is launched into two $3 \mathrm{~dB} 2 \times 2$ multimode fiber couplers, which splits each beam into two equal signals. One beam propagates along the lead-in fiber to the sensor head, while the other is terminated at the end-face of one of the coupler's output fibers. The reflected signal from the sensor head also propagates along the same fiber to the same coupler, and then both of the light beams are detected by the fiber optic spectrometer. A multimode white light interferometric spectrum analyzer is used to obtain the flow rate by demodulating the light signal fringes from the detected spectrums. 


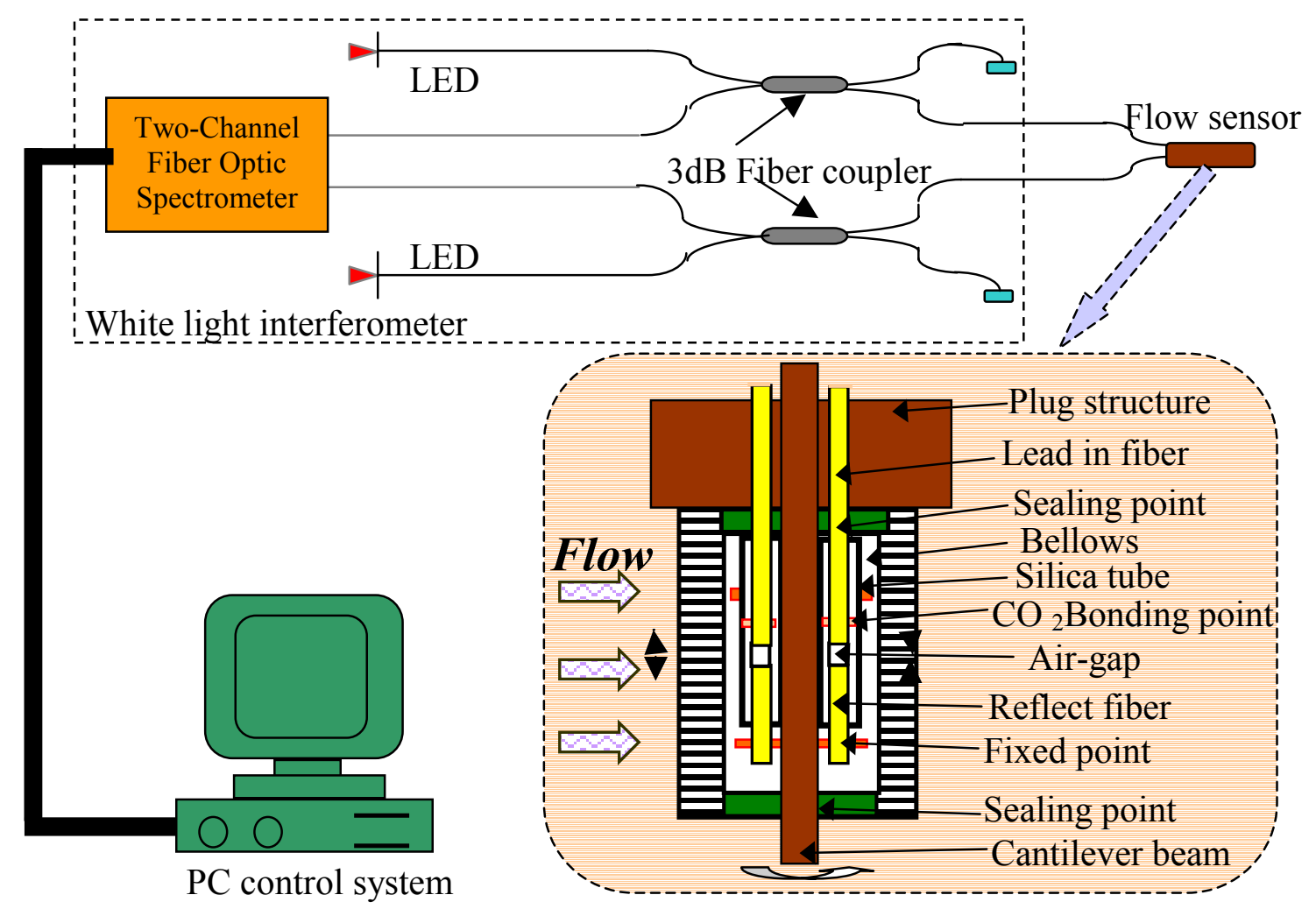

Figure 3.1. Fiber optic flow sensor system.

The configuration of the fiber optic flow sensor head shown in Figure 3.1 is a combination of the broadband white light interferometer and cantilever beam designs. Based on the strain principle of cantilever beams ${ }^{1}$, the flow rate can be determined by detecting the air-gap changes from two fiber sensors caused by the bending of the cantilever beam. The sensor head is fabricated by bonding two identical fiber optic tube-based sensors on a cantileverbeam. Each sensor is fabricated by inserting two fibers into a fused silica hollow glass tube. The process is somewhat different from normal tube-based fiber optic sensor fabrication ${ }^{2}$, because while one end of the capillary tube is thermally bonded to the lead-in fiber, the reflecting fiber is left free to move in the other end of tube. Therefore, an air gap is formed between the two cleaved fiber end faces. The sensor fabrication process is then repeated for the other side of the beam. Both ends of the tube and both reflecting fibers are firmly bonded to the metal cantilever beam at a predetermined gauge length. This allows the air gap to change freely without restriction by the capillary tube and only related with the changes in bending of the cantilever beam caused by the flow rate of the fluid. This dual sensor combination is then inserted into the central hole of a metal tube or plug, which allows easy installation of the sensor plug into the flow pipes for measurement. Finally a small metal bellows is used to cover the beam and sensors. This bellows allows the flow to bend the cantilever beam located inside without allowing the fluid to contact the cantilever beam or sensors. In this manner, the sensor can be isolated from the fluids (which may be corrosive) in the outside environment. When the fluid flows past the sensor, the cantilever beam will be bent by the force caused by the momentum of the fluid passing the beam. 
When a fluid impinges on the beam, all its momentum can be considered converted into force, which can be expressed as

$$
F \times t=m \times v
$$

where $F$ is the force on the cantilever, $t$ is the unit of time, $v$ is the velocity of the fluid, and $m$ is the quantity of the passing fluid within the unit time. These quantities are related as follows.

$$
\left\{\begin{array}{c}
m=\rho \times V \\
V=S \times v \times t \\
S=c \times L
\end{array}\right.
$$

where $\rho$ is the density of the fluid, $V$ is the volume of flow in unit time; $S$ is the area of the cantilever beam, $c$ is width of cantilever, $d$ is thickness of cantilever, $L_{b}$ is the length of the cantilever beam. From Eq. (3-1) and (3-2) the applied force per unit time $t$ is:

$$
F=\rho \times c \times L_{b} \times v^{2}
$$

Suppose $F$ is a uniform force, the maximum strain $\varepsilon$ will be at the fixed end of the cantilever

$$
\varepsilon=\frac{6 F L_{b}}{E c d^{2}}
$$

where $E$ is theYoung's modulus. When the flow passes by, because each of the sensor's free fibers are bonded to cantilever, bending of the beam will be manifested as an air gap change. The air gap variation caused by the gauge length change $\Delta L$ between the two bonding points can be represented as

$$
\Delta L=\varepsilon \times L
$$

where $L$ is the gauge length between two bonding points. Then from Eq. (3-2) to (3-5), we obtain

$$
\Delta L=\frac{6 \rho v^{2} L_{b}^{2} L}{E d^{2}}=\beta(v)
$$

where $\beta$ denotes the relationship between the air-gap change $\Delta L$ and the velocity of the flow $v$.

When the cantilever-beam bends due to the flow of the fluid, the air-gap of the sensor on the side facing the fluid flow will increase, while the air-gap of the sensor located on the opposite side of the cantilever beam will decrease. For a given material, the volume compressibility and thermal expansion should be approximately linear for small pressure and temperature changes. Therefore, when fluid passes by the sensor, the air gap of both sensors will change but in opposite directions. However, both sensors will have the same magnitude and direction of air gap change due to the temperature and pressure changes:

$$
\left\{\begin{array}{l}
\Delta L_{1}=a_{1} \Delta T+K a_{1} \Delta P+\beta_{1}(v) \\
\Delta L_{2}=a_{2} \Delta T+K a_{2} \Delta P+\beta_{2}(v)
\end{array}\right.
$$


where $\Delta L_{1}, \Delta L_{2}$ represent the gauge length changes of the two sensors, $\alpha_{1}, \alpha_{2}$ are the temperature effects of the two sensors, $K$ is the relationship between the effects of thermal extension and pressure extension, and $\beta_{1}, \beta_{2}$ refer to the flow coefficients of the two sensors as shown in Eq.(3-6). We can cancel the effects from temperature and pressure based on the above equations to obtain the relationship $F$ between the flow rate and system output based on the two air-gap changes.

$$
F(v)=a_{2} \Delta L_{1}-a_{1} \Delta L_{2}=a_{2} \beta_{1}(v)-a_{1} \beta_{2}(v)
$$

From Eq. (3-8), we can see that by analyzing the outputs of the two sensors, this sensor system can detect the applied flow velocity while being insensitive to pressure and temperature changes by automatically compensating for temperature and pressure effects. If we select the cantilever-beam to be brass with $E=4.2 \times 10^{10} \mathrm{~kg} / \mathrm{m}^{2}, L_{b}=25 \mathrm{~mm}, c=5 \mathrm{~mm}$, $d=0.5 \mathrm{~mm}$, and the two fiber sensors with gage length of $L=20 \mathrm{~mm}$, original air gap of a= $12.00 \mathrm{~mm}$, then the relationship between the air gap changes and the applied flow velocity can be calculated as shown in Figure 3.2. According to this simulation, we can see that when the cantilever beam temperature is changed, both the front and back sensor air gaps are affected, causing the relationship between sensor output and the applied flow rate very repeatable, which illustrates that the sensor can compensate for temperature automatically.

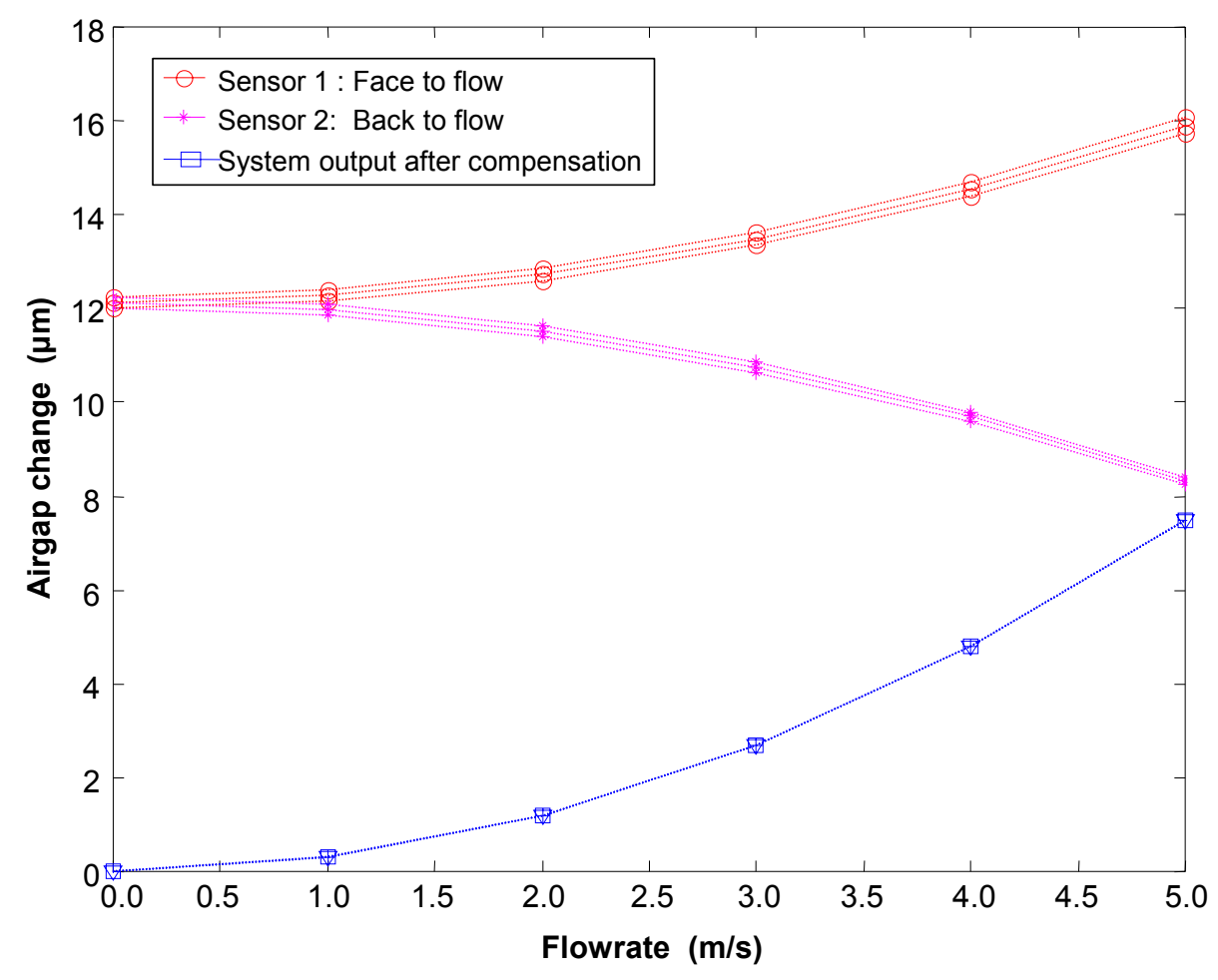

Figure 3.2. Simulation of flow sensor self-compensation.

In industrial practice, flow measurements are usually used to obtain the flow rate rather than the flow velocity, so the related cross sectional area of the flow pipes must be added to Eq. (3-8) to obtain the relationship between the air gap changes and flow rate. It is also possible 
that the relationship between air gap changes and flow velocity will differ from the theoretical value due to materials processing and fabrication techniques, but these factors can all be combined within Eq. (3-8) and will be adjusted for during system calibration.

\subsection{Flow Sensor Construction}

The prototype flow sensor system was constructed as shown in Figure 3.1. A dual channel white light interferometer system was used for optoelectrical signal processing. Two Honeywell 4854 LEDs with center wavelengths around $850 \mathrm{~nm}$ pigtailed with standard 50/125 multimode fibers were used as broadband light sources. A PC-2000 dual-channel fiber optic spectrometer from Ocean Optics, Inc. was used to detect the optical signals from the sensor head, and its output fed to the flow rate measurement control system based on a multimode white light interferometric spectrum analyzer.

The analysis described in the previous section provides a clear guide to the design of the fiber optic sensor for flow detection. The fabrication technique was gradually improved through experience after making many sensor heads. Due to Invar's low coefficient of thermal expansion $(\mathrm{CTE})\left(1.05 \times 10^{-6} /{ }^{\circ} \mathrm{C}\right)$, which is much lower than most conventional metals and closer to fused silica ${ }^{3}$, an Invar sheet from Electronic Space Products International, Inc was selected for the cantilever-beam and cut, polished and annealed. The inner diameter of the hollow fused quartz tubing was chosen to be $132 \mathrm{~mm}$, which limits the movement of the multimode fiber $(125 \mathrm{~mm})$ to $5 \mathrm{~mm}$. PN-231 regular Nickel bellows from Servometer, Inc. were used for the packaging of the flow sensor. The size of the Invar cantilever beam was selected as $3 \mathrm{~mm} \times 30 \mathrm{~mm}$. Based on the working range and resolution of the multimode white light system ${ }^{4}$, the thickness of the invar sheet, the gauge lengths and the original air-gaps of both fiber sensors were adjusted to maximize the visibility of both sensors through the complete measurement range. Also the gauge length was selected to be as small as possible to decrease the inherent thermal expansion. In fiber fabrication, the initial air gap between both fiber end-faces was adjusted to obtain the highest interference fringe visibility. The fabrication process is shown in Figure 3.3a and b; the photograph of the finished flow sensor is shown in Figure 3.3c; the sensor plugged into its fitting for testing or measurement is shown in Figure 3.3d. Both the lab-scale testing and field-testing have been performed to evaluate the prototype flow sensor system, as described in the following sections. 


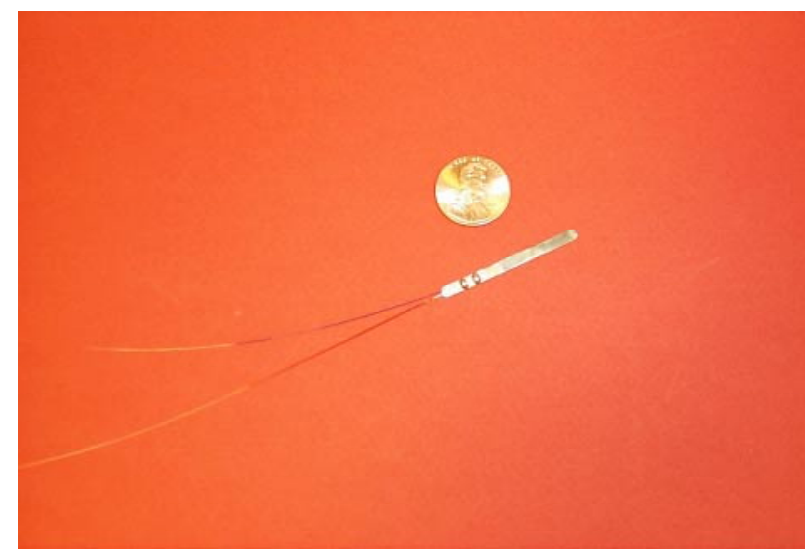

(a) Two symmetrical fiber ferrule sensors fabricated and bonded at two sides of Invar cantilever beam

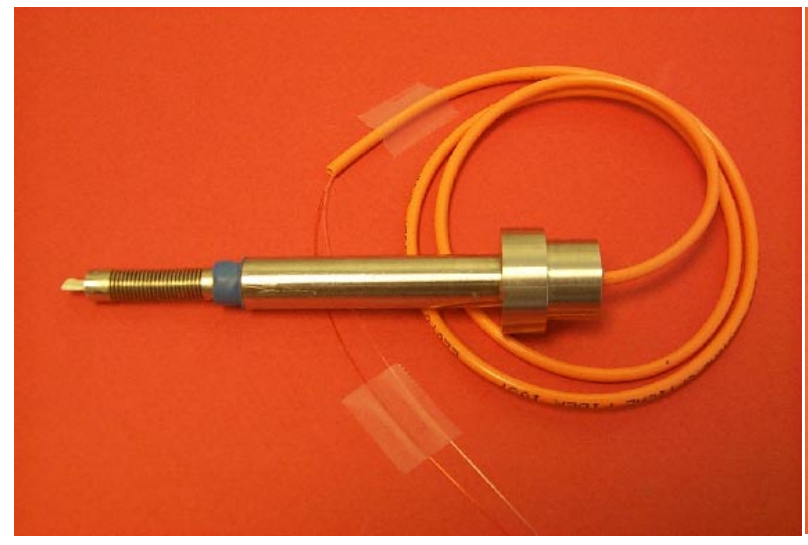

(c) Prototype fiber optic flow sensor

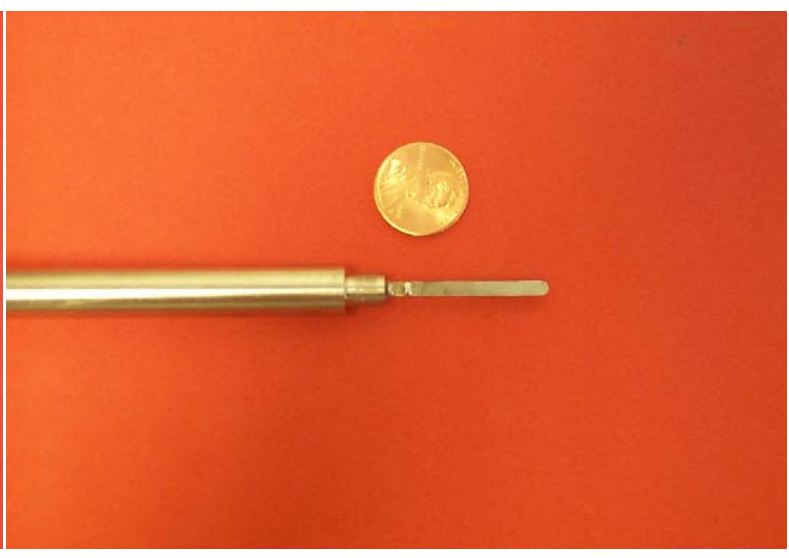

(b) Flow sensor fixed in packaging structure

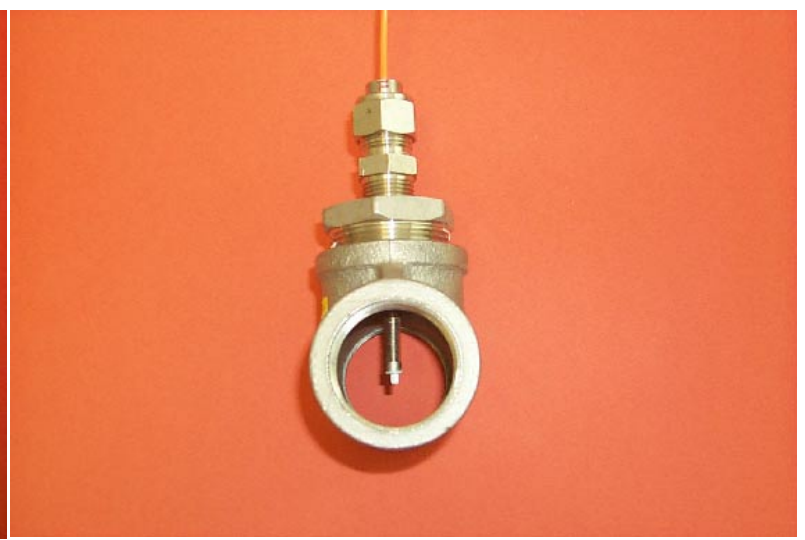

(d) Flow sensor fixed into T-fitting

Figure 3.3. Fabrication of flow sensor.

\subsection{Lab-Scale Experiments and Results}

Evaluation and testing of the flow sensors is necessary to improve the flow sensor design and fabrication. The lab-scale test system was set up as illustrated in Figure 3.4. This experiment set-up includes a FT-24NENW turbine flow meter, purchased from Flow Technologies Inc. For 1-1/2" pipe sizes, the custom ordered calibration flow rate range is from 1.6 to $90 \mathrm{GPM}$, the repeatability is $\pm 0.05 \%$ of the full range, and the linearity is $\pm 0.5 \%$ of reading. A $1 / 4 \mathrm{HP}$ Wayne submersible utility pump was used to drive the water through the PVC pipes; with 11/2"pipe, the maximum flow rate should be 18.5GPM. A DPF700 meter from Omega, Inc was ordered with a RS-232 option board to connect to a laboratory computer for preliminary flow rate testing. Both the fiber optic sensor and the thermometer were put into the testing pipes, and the pumped flow rate adjusted by changing the input of the electrical pump. Both the flow sensor output and the flow meter output were recorded for comparison. The testing system at CPT is shown in Figure 3.5. 


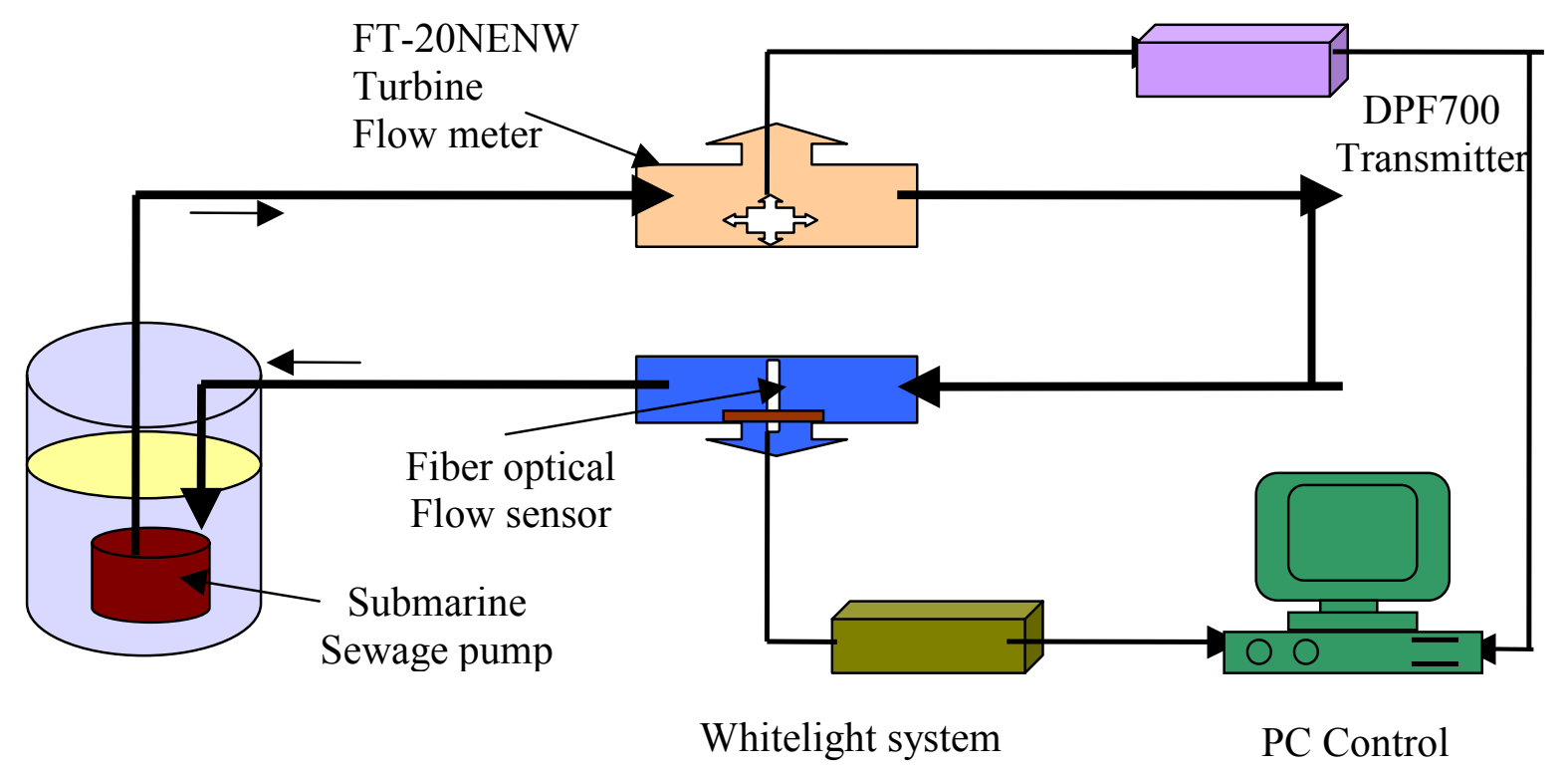

Figure 3.4. Lab-scale flow testing system setup.

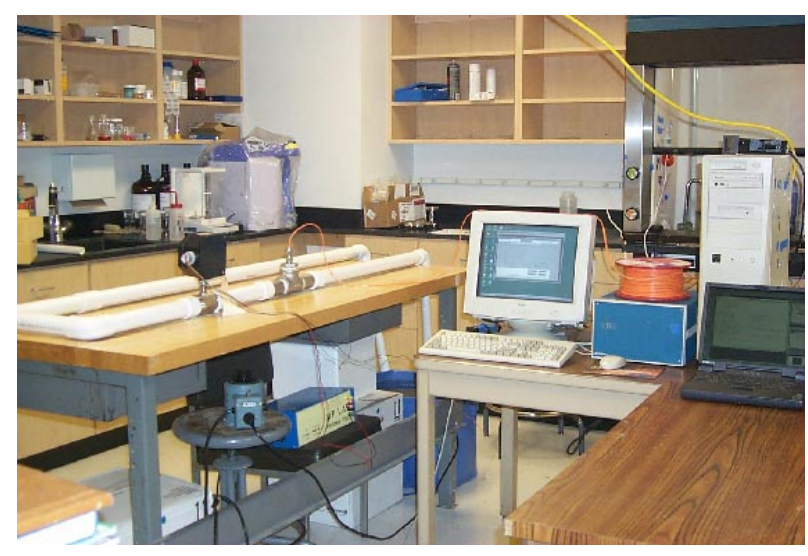

(a) Lab-scale flow testing system loops

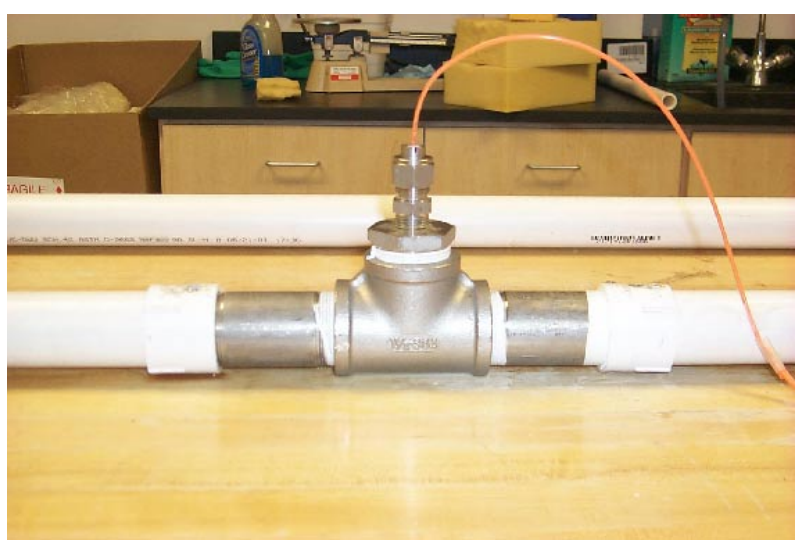

(b) Flow sensor deployed in testing

Figure 3.5. Flow sensor testing setup in Center for Photonics Technology, Virginia Tech.

Using this testing system, numerous experiments have been carried out to evaluate the performance of the designed flow sensor system. The normal test temperature range was $18 \sim 45^{\circ} \mathrm{C}$ with normal atmospheric pressure. The results shown below were obtained using the fiber optic flow sensor whose parameters are shown in Table 1. 
Table 3.1. Parameters of fiber optic flow sensor used for lab scale testing.

\begin{tabular}{ll}
\hline Fiber type: & Multimode $50 / 125$ \\
Cantilever beam material: & Invar sheet \\
Cantilever beam size: & $0.010^{\prime *} 1.5^{\star} 0.13^{\prime \prime}$ \\
Original air gaps of two sensors: & $9.211 \mu \mathrm{m} ; 8.810 \mu \mathrm{m}$ \\
Original visibility of two sensors: & $67 \% ; 69 \%$ \\
Gauge length of two sensors: & $1.8 \mathrm{~mm} ; 2 \mathrm{~mm}$ \\
Relative temperature coefficients: & $0.9 ; 1$ \\
Sensor protection: & Flexible Ni-bellows \\
\hline
\end{tabular}

\subsubsection{Sensor calibration}

Sensor calibration is very important for sensor evaluation and application. It is usually conducted by applying known flow rates within its operating range to obtain the calibration curve, or one-to-one relationship between the original sensor output and the applied flow rate, which can be stored in the host computer and later used to convert sensor output to the flow rate reading. To ensure the calibration accuracy, the flow rate was held at each step for about three minutes before moving to the next step during the calibration process. By averaging the output of both the flow meter and flow sensor at each step, errors can be minimized. The original data is shown in Figure 3.6. Because the temperature effect coefficients (TEC) were determined by testing prior to sensor calibration (Table 3.1), Eq. (31) (3-3) were used to cancel the temperature effects. The compensated output is shown in Figure 3.6 and used to obtain the flow sensor calibration curve shown in Figure 3.7. In general, the calibration curve was obtained by taking the average of several consecutive sets of calibration data to further ensure the calibration accuracy and to generate the coefficients of calibration.

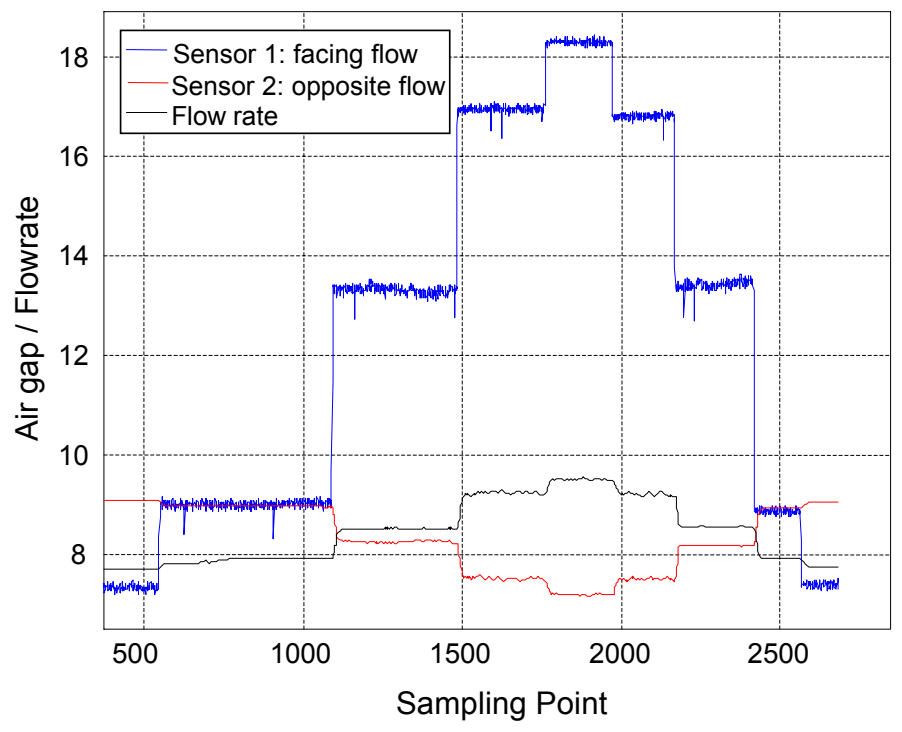

Figure 3.6. Relationship between flow rate and flow sensor air gaps. 


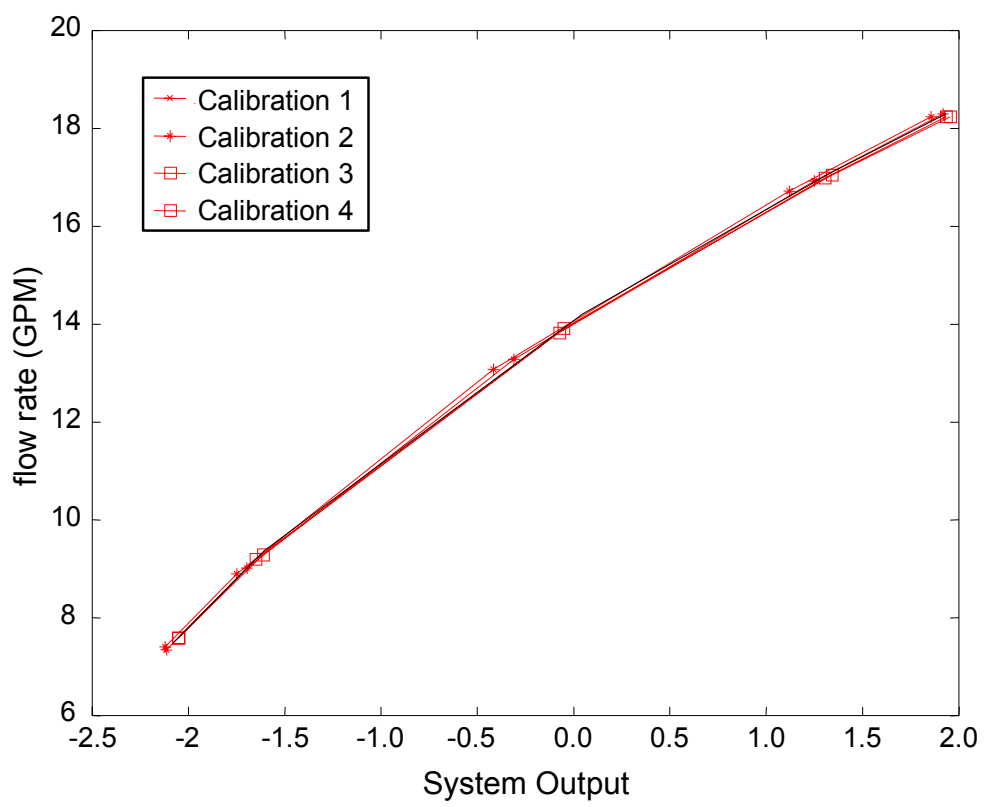

Figure 3.7. Flow sensor calibration curve.

\subsubsection{Temperature compensation}

Temperature dependence is critical for flow sensors used in oil field environment. It is a great challenge to design a flow sensor to have minimum (ideally zero) temperature dependence. With the self-compensation design as described in Section 2, it is possible, in theory, to have very small or even zero temperature sensitivity. Figure 3.8 shows the temperature independence characteristic of the flow sensor. Even though the environmental temperature changes from $23^{\circ} \mathrm{C}$ (squares) to $45^{\circ} \mathrm{C}$ (stars), the output of the fiber flow sensor matched the applied flow rate with high repeatability, indicating that this sensor design can compensate the temperature changes automatically, consistent with the theoretical analysis.

\subsubsection{Measurement result}

Using the calibration curve or coefficients, the fiber optic sensor was used for flow rate measurement and the results compared with the flow rate output from FT-24NENW flow meter in Figure 3.9. 


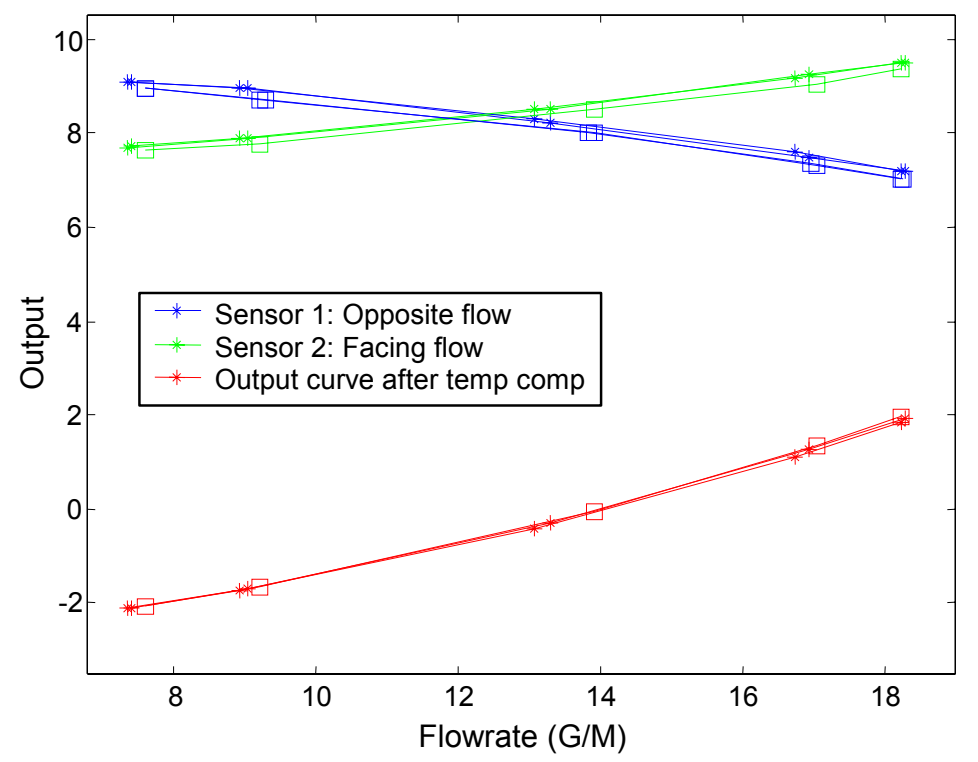

Figure 3.8. Fiber optic flow sensor self-compensation result.

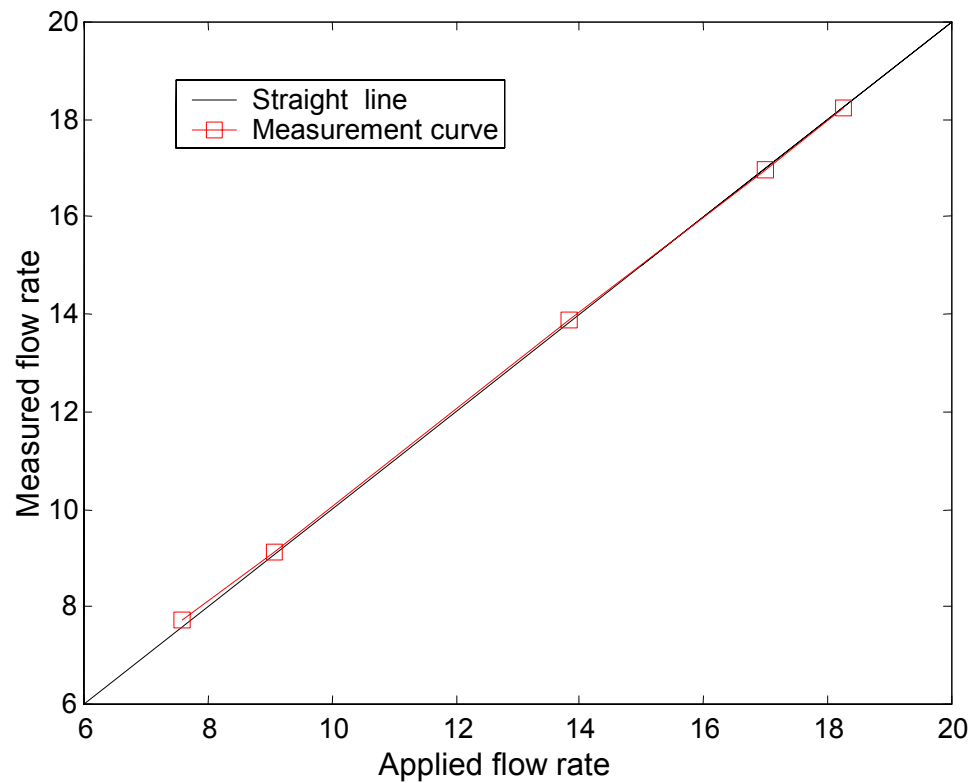

Figure 3.9. Flow sensor system calibration lines.

\subsubsection{Repeatability}

Sensor repeatability can be determined by applying the a preset flow rate repeatedly from one direction (increasing or decreasing). The largest difference in the sensor output readings can be used to specify the repeatability of the sensor. Under different water temperatures, four measurements up to the full operating range of the sensor were performed with the results shown in Figure 3.10a. For comparison purposes, the original calibration data is also shown. 
The deviation of the two measurements with respect to the calibration data is plotted in Figure 3.10b. The maximum deviation between the measured pressure and the calibrated pressure was within $\pm 0.3 \mathrm{GPM}$. The normalized repeatability of the sensor system with respect to its dynamic range was therefore $\pm 1.62 \%$ of the full scale. This test also demonstrates that this sensor design can effectively compensate for temperature influences.

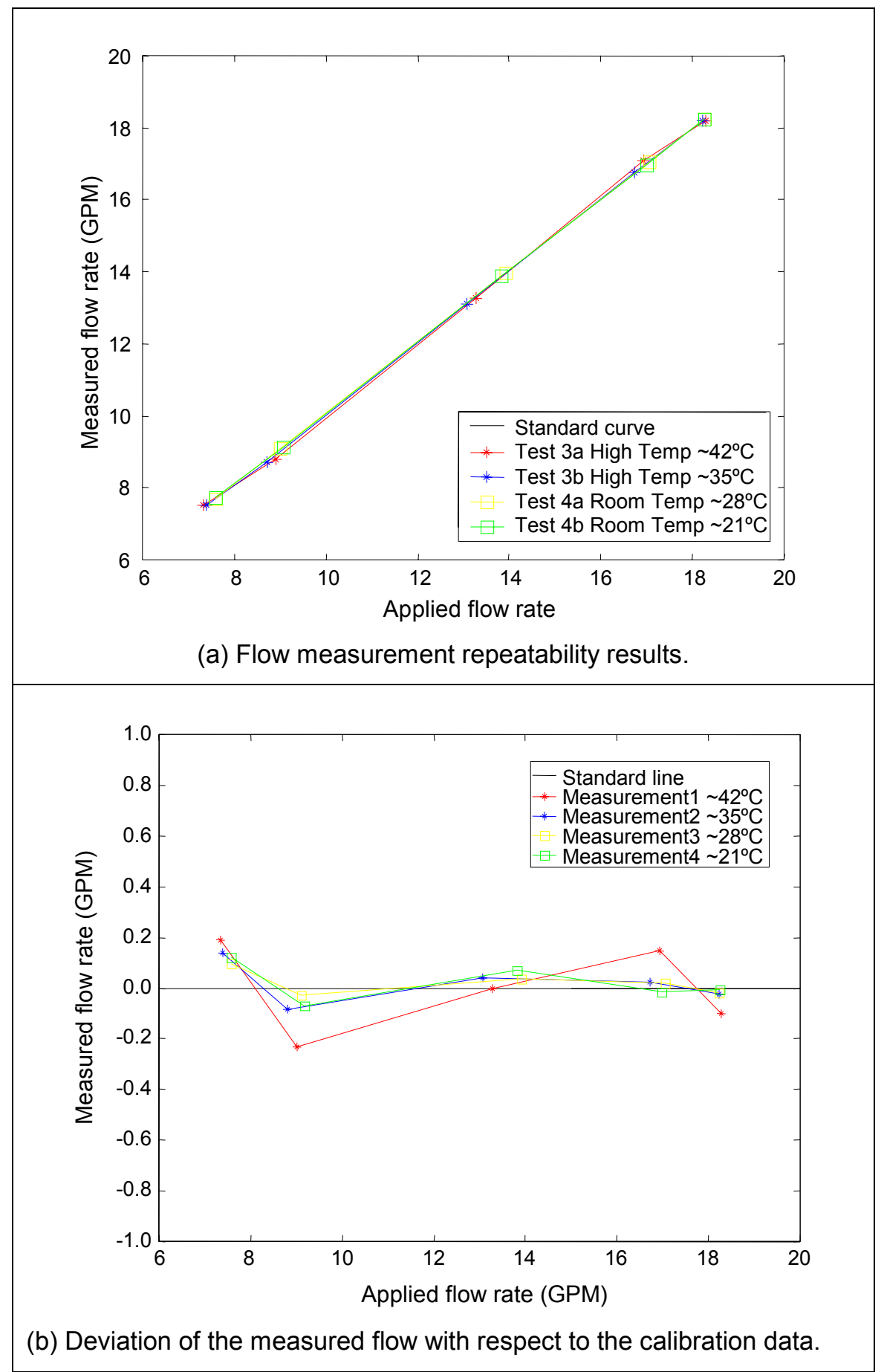

Figure 3.10. Flow measurement repeatability test results. 


\subsubsection{Dynamic flow measurements}

To demonstrate the sensor's dynamic response, the calibrated flow sensor was applied to dynamic flow measurements. The reference flow signal was provided by the FT-24NENW Turbine flow meter. Comparison of the output of both the fiber flow sensor and flow meter shown in Figure 3.11 reveals that the fiber sensor follows the turbine flow meter very well.

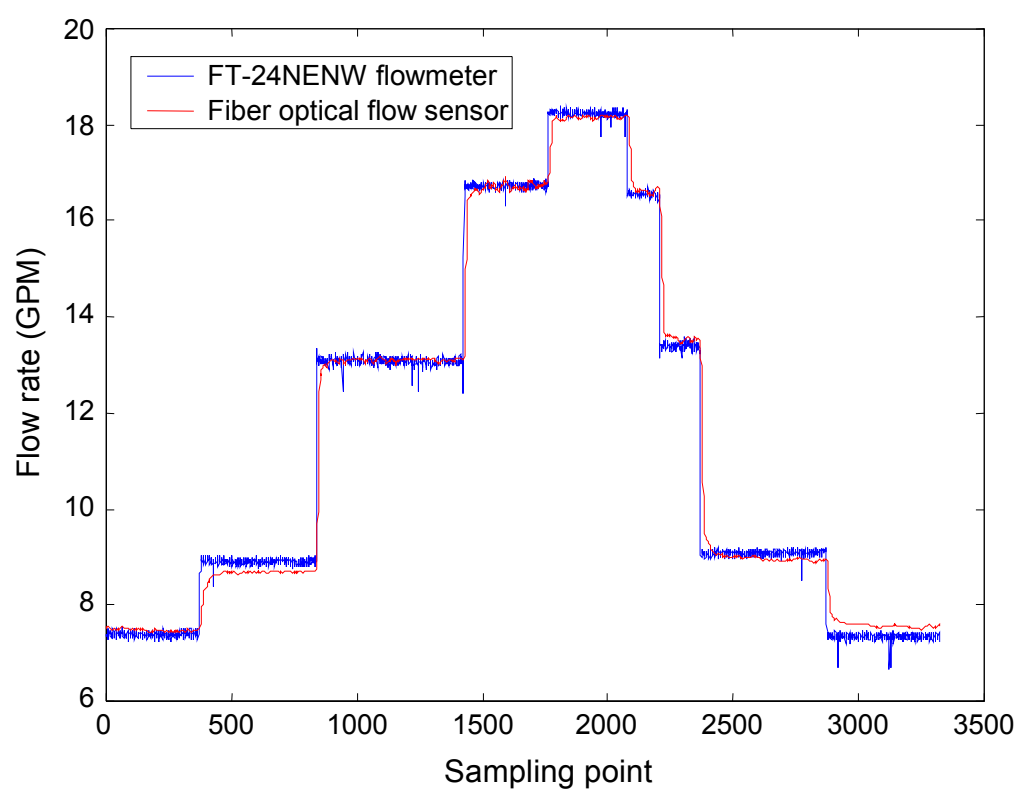

Figure 3.11. Dynamic response of flow sensor.

\subsubsection{Resolution of flow rate measurement}

The resolution of a sensor system is usually interpreted as the standard deviation of a series of flow measurements. It is common to use twice the standard deviation as the direct measure of resolution. Evaluation of the flow sensor resolution was performed using a calibrated sensor. The sensor was placed the testing pipe and the data from the test system was sampled at a rate of 60 samples per second for one minute, which should be sufficient compared to the system's frequency response of $10 \mathrm{~Hz}$. The flow rate measurement outputs within the oneminute sampling period are plotted in Figure 3.12. The standard deviation of the flow data within this time period was calculated to be $\sigma=0.0097 \mathrm{GPM}$. Therefore the resolution of the sensor system was estimated to be $2 \sigma=0.0194 \mathrm{GPM}$. The normalized resolution with respect to the dynamic range of the system was $0.11 \%$ of full scale. 


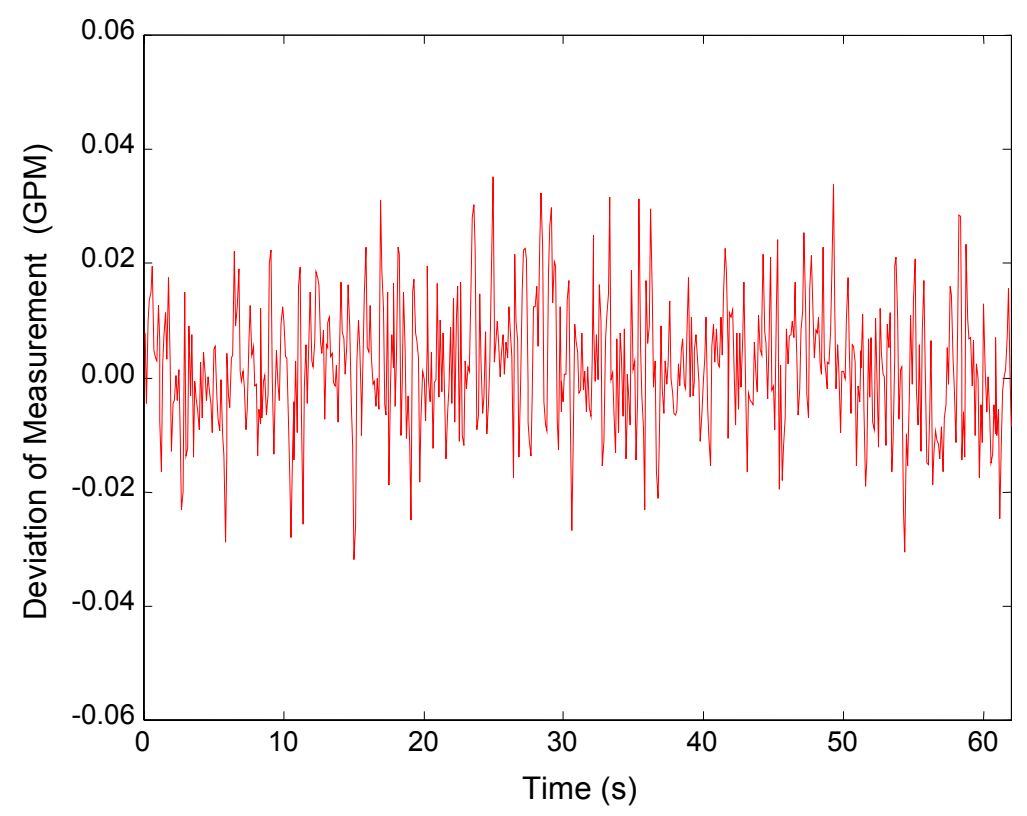

Figure 3.12. Resolution of flow sensor system.

\subsubsection{Hysteresis of flow rate measurement}

The hysteresis of the flow sensor can be measured by cycling the applied flow between the minimum and the maximum of the operating range in both increasing and decreasing directions. The hysteresis can be calculated as the largest difference between the output readings of the pressure cycles. Experiments were conducted to evaluate the actual hysteresis of the flow sensor probe. The evaluation was conducted using the same flow testing system after the sensor system was calibrated. The applied flow was first increased to the maximum operating range of $18.5 \mathrm{GPM}$, and then decreased after it was maintained at $18.5 \mathrm{GPM}$ for 30 minutes. The experimental results shown in Figure 3.13 demonstrate that the hysteresis of the full operating range is less than $1.35 \%$. 


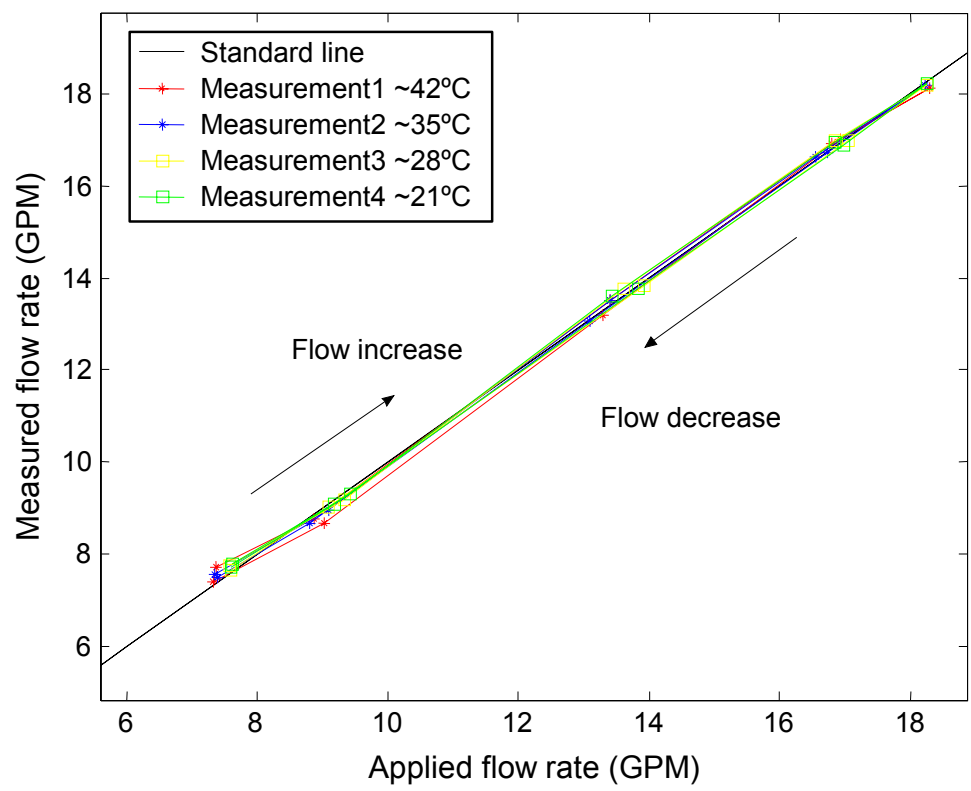

(a) Flow measurement hysteresis test results

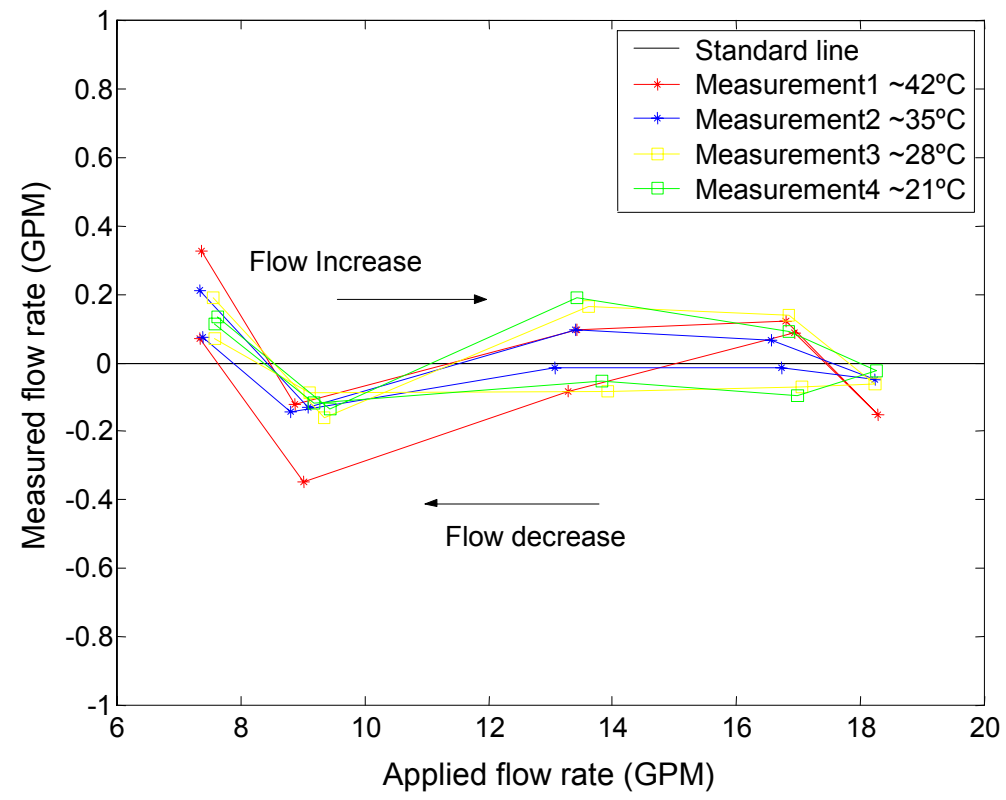

(b) Deviation of the measured flow with respect to the calibration data

Figure 3.13. Hysteresis of flow sensor in lab-scale testing. 


\subsubsection{System stability test}

The same flow sensor was also used to test the system stability. The sensor was kept in the flow-testing loop for 12 hours while the flow was maintained at 7.2GPM. The data acquisition system was programmed to sample the sensor's output every 10 seconds. The test results are shown in Figure 3.14. Processing the data revealed that the maximum peak-topeak pressure variation within the 12-hour time period was $0.35 \mathrm{GPM}$. The data also showed that the output from FT-24NENW flow meter exhibited many discontinuities and fluctuations, which limited the evaluation of flow sensor performance. Thus we believe that the maximum variation was less than $1.89 \%$ of the full dynamic range.
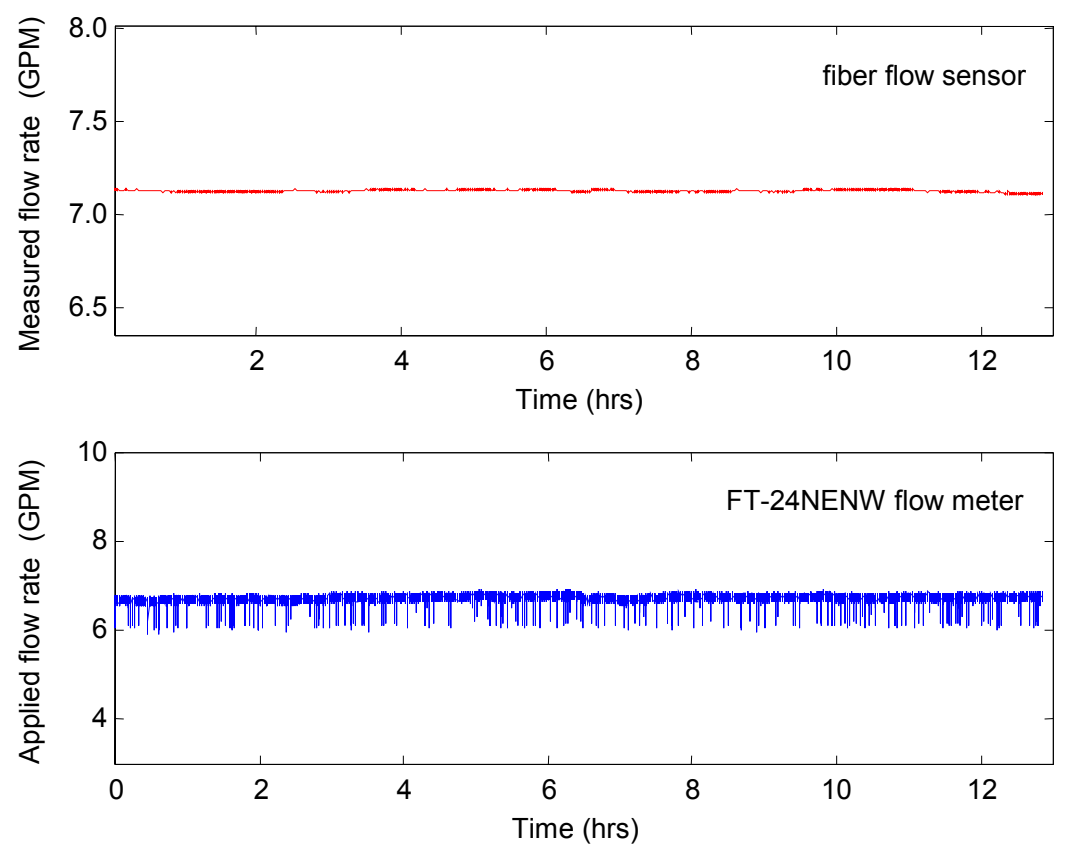

Figure 3.14. Flow sensor system stability test over 12 hours at 7.2 GPM. 


\subsection{Field Testing of Flow Sensor System}

To demonstrate the feasibility of the developed flow sensor system, the prototype sensor system was field tested at the flow loops at the University of Tulsa from Aug 19, 2002 Aug 23, 2002. Two flow loops were involved in this field-tests; the detailed technical parameters and testing results are reported here.

\subsection{Indoor Flow Testing}

The indoor flow facility we used for flow testing is shown in Figure 4.1a. A variable frequency pump (0 1745RPM) from Robins \& Meyers, Inc (Figure 4.1b) was used to pump the water into 1-1/2" Schedule 40 flow loops, which combine many gauges including an adjustable pressure valve, pressure gauge from Wika, Inc and thermocouple from Omega, Inc. As shown in Figure 4.1(c), the flow sensor was deployed in the flow pipes using a $0.5 \sim 1.5$ inch NPT bushing through a 1.5inch NPT T-fitting. Figure 4.1(d) shows the flow sensor connected by a dual fiber cable to the rest of the fiber optic flow measurement system, which mainly includes a multimode fiber optic white light interferometric system. The output of white light system is shown as Figure 4.1(e), the flow rate measurement window is shown as Figure 4.1(f). The temperature of the flow loops during indoor testing was controlled by the air conditions and was stable at around $29^{\circ} \mathrm{C}$; the pressure in the flow pipes was controlled at around 5psi. The parameters of fiber optic flow sensor used in this indoor testing are listed in Table 4.1.

Table 4.1. Parameters of fiber optic flow sensor used for indoor flow testing in Tulsa.

\begin{tabular}{ll}
\hline Fiber type: & Multimode $50 / 125$ \\
Cantilever beam material: & Invar sheet \\
Cantilever beam size: & $0.010^{\prime *} 1.5^{\star} 0.13^{\prime \prime}$ \\
Original air gaps of two sensors: & $10.46 \mu \mathrm{m} ; 11.96 \mu \mathrm{m}$ \\
Original visibility of two sensors: & $71 \% ; 21 \%$ \\
Gauge length of two sensors: & $2.2 \mathrm{~mm} ; 1.8 \mathrm{~mm}$ \\
Relative temperature coefficients: & $1 ; 0.85$ \\
Sensor protection: & Flexible Ni-bellows \\
Test conditions: & $84 \sim 86^{\circ} \mathrm{F} ; 1 \sim 5 \mathrm{psi}$ \\
\hline
\end{tabular}




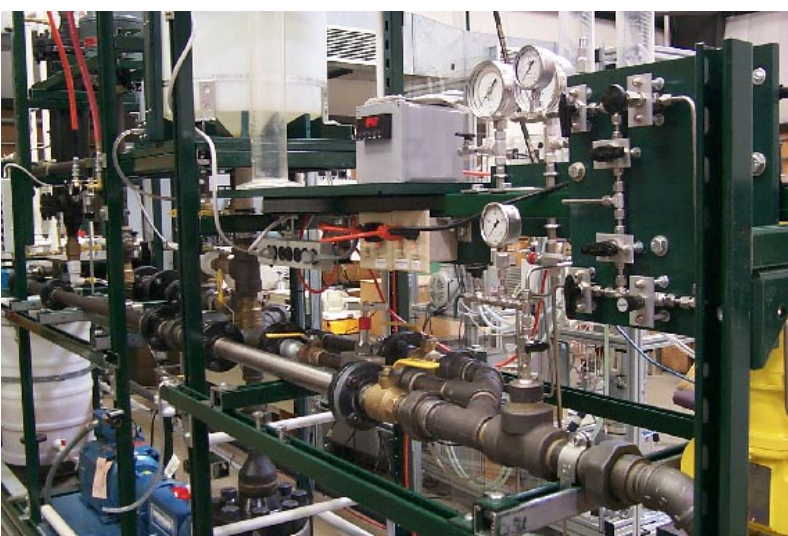

(a) Indoor flow test system in Tulsa University, OK

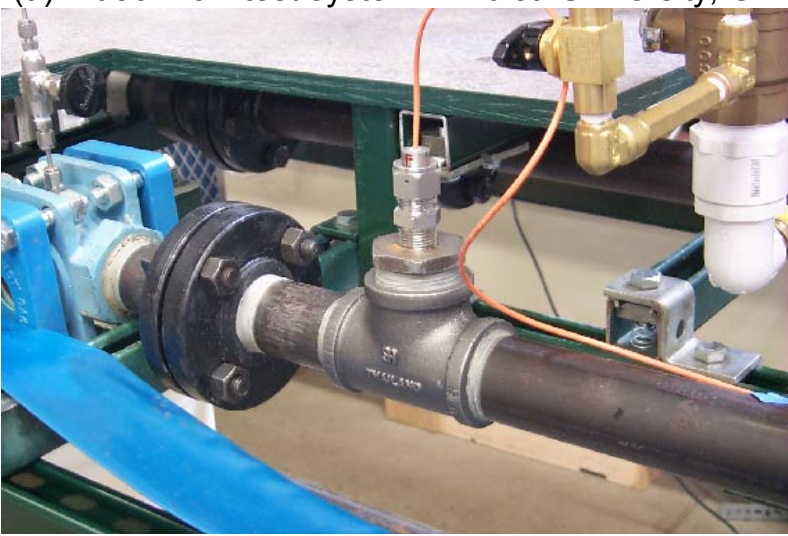

(c) Flow sensor in indoor flow loop

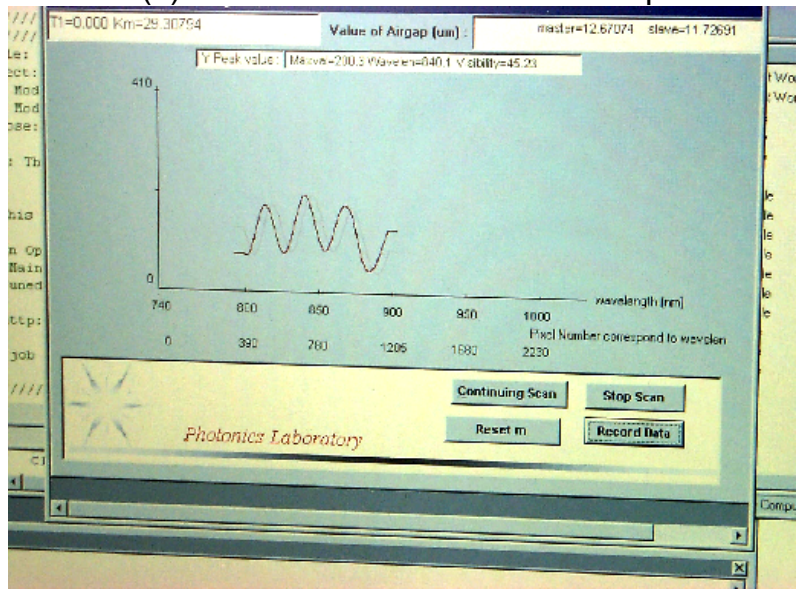

(e) Fiber optic white light system output window

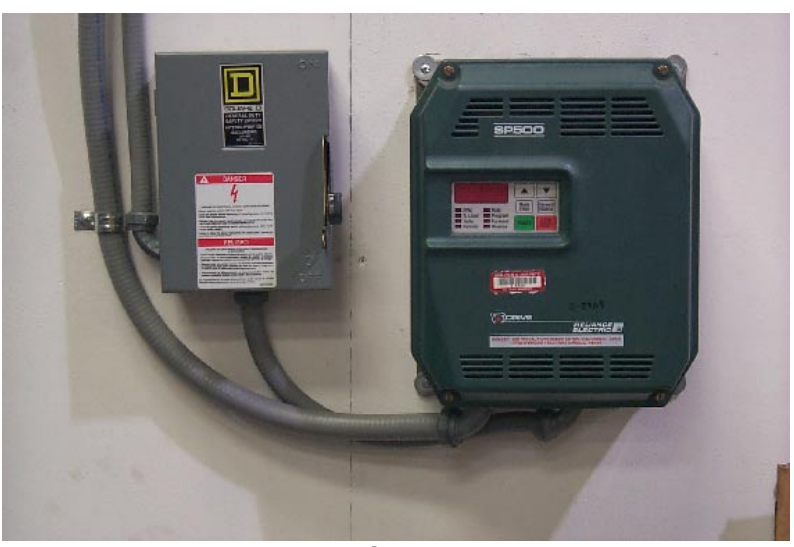

(b) Variable frequency pump

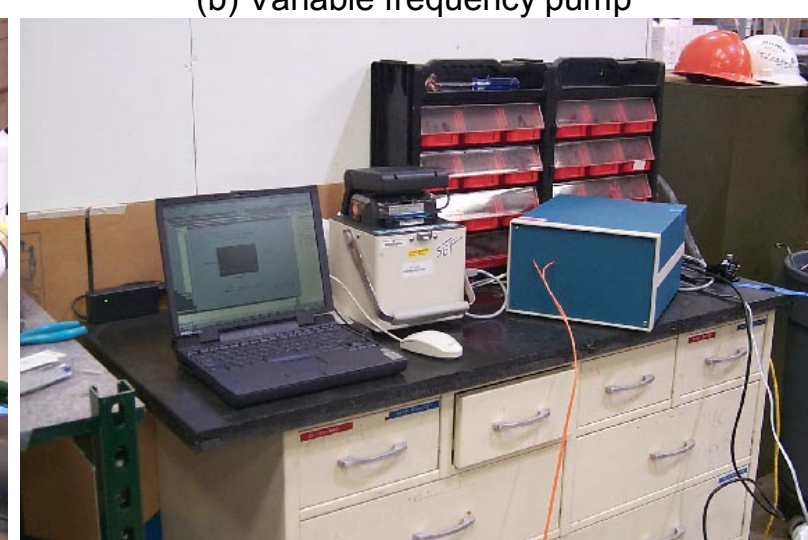

(d) Flow sensor system in indoor flow testing room

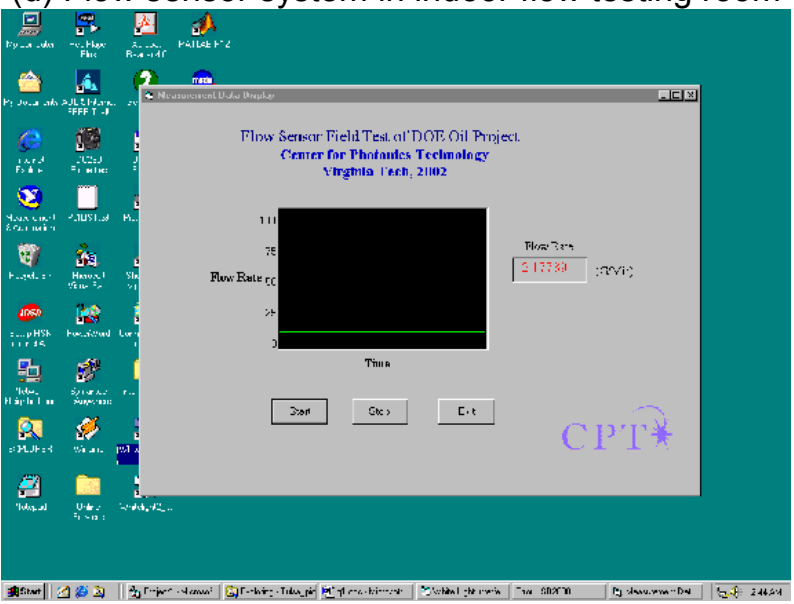

(f) Flow sensor system output window

Figure 4.1. Indoor flow testing system in University of Tulsa, OK.

Compared with the outdoor flow loops, the indoor flow testing facilities were at a very stable temperature and pressure. Due to some unexpected occurrences during shipping, when we arrived at the Tulsa testing field, only one channel of the white light system could be used for the indoor testing while we completed an urgent repair of the other channel. So for indoor testing, we used only one of the two sensors to measure the indoor loop flow rate. 


\subsubsection{Variable frequency pump calibration}

The indoor flow testing system used a variable frequency pump from Robins \& Meyers, Inc, which had an operating range from 0 to1745RPM, to drive water in the flow loop. Using the FT-NENW turbine flow meter, a calibration was performed first to convert the flow rate units from RPM to GPM; the calibration curve was shown in Figure 4.2.

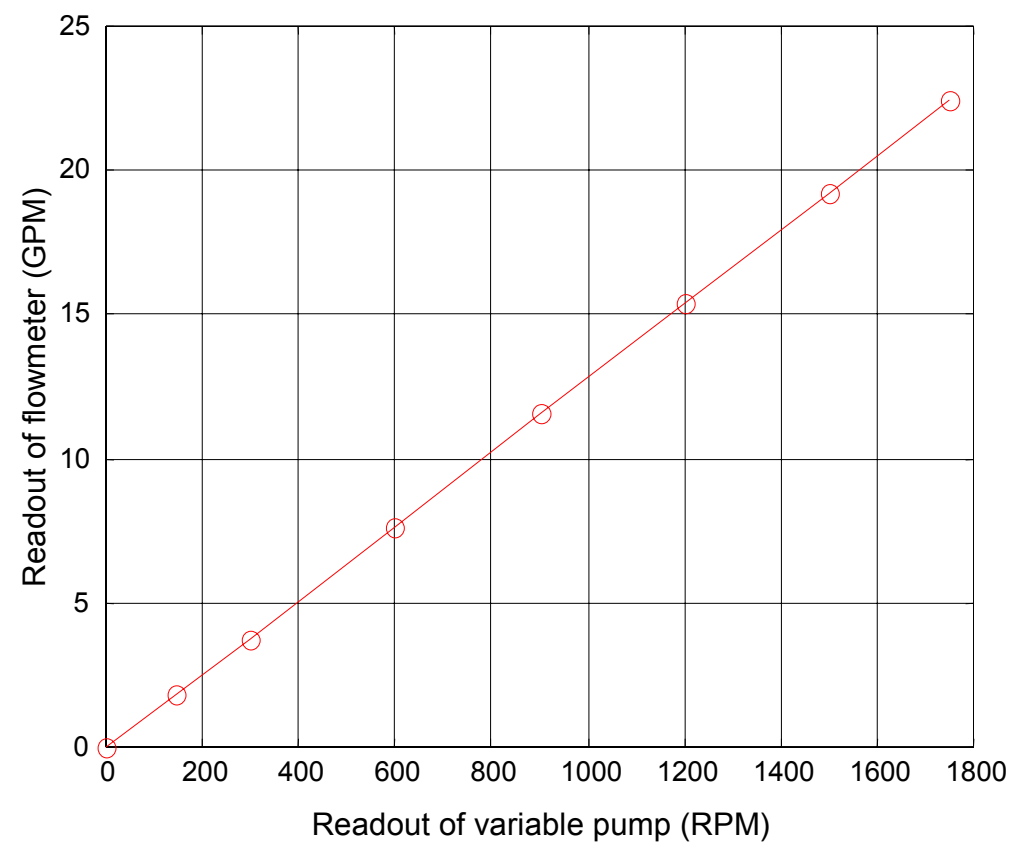

Figure 4.2. Variable frequency pump calibration curve using FT-24NENW flow meter.

\subsubsection{Sensor calibration}

Due to the difference in testing conditions between the indoor test loop and lab-scale testing, the sensor required recalibration prior to the testing in the outdoor system. The calibration curve of the fiber flow sensor using the output from a reference flow rate is shown in Figure 4.3. 


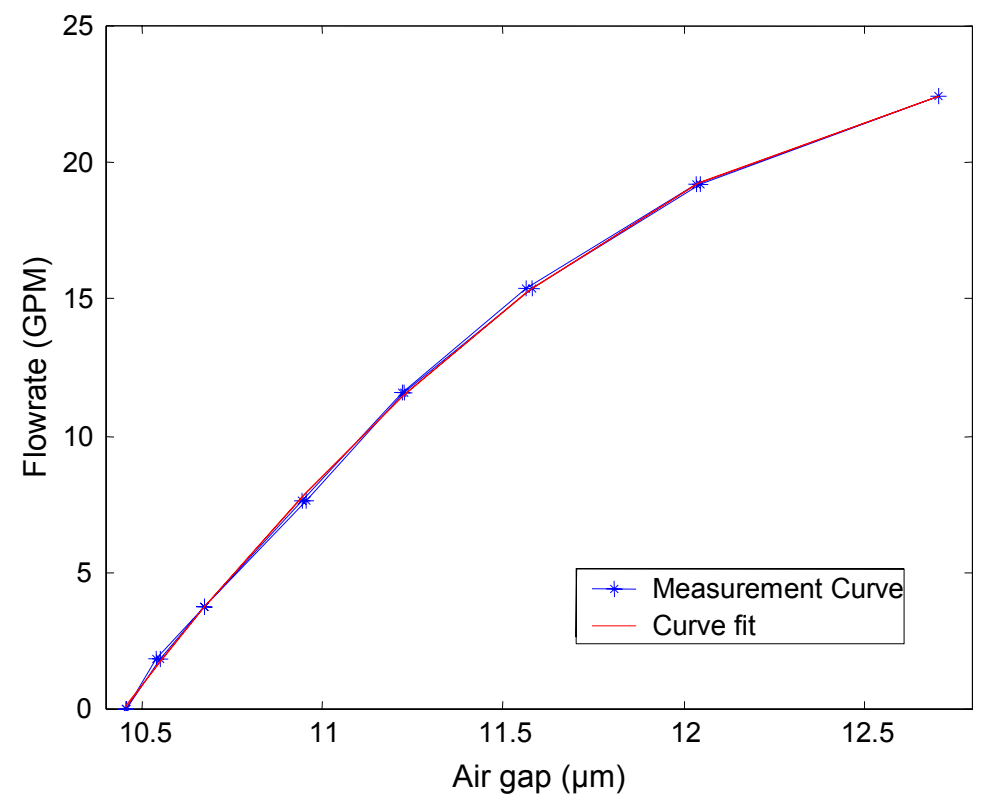

Figure 4.3. Flow sensor calibration curve for indoor flow testing in Tulsa, OK.

\subsubsection{Measurement results and repeatability}

The real-time flow rate information was obtained through the calibrated relationship between the reference flow rate and the airgap change in the sensor head. Figure 4.4a shows the measurement results for repeatability testing. The flow sensor output is a linear function of the variable frequency pump rotational speed. A magnified view of the repeatability results is shown Figure $4.4 \mathrm{~b}$. For the full measurement range from 0 to 22.42 gallons per minute (GPM), the maximum deviation was $\pm 0.26 \mathrm{GPM}$, which is $\pm 1.15 \%$ of full scale. 


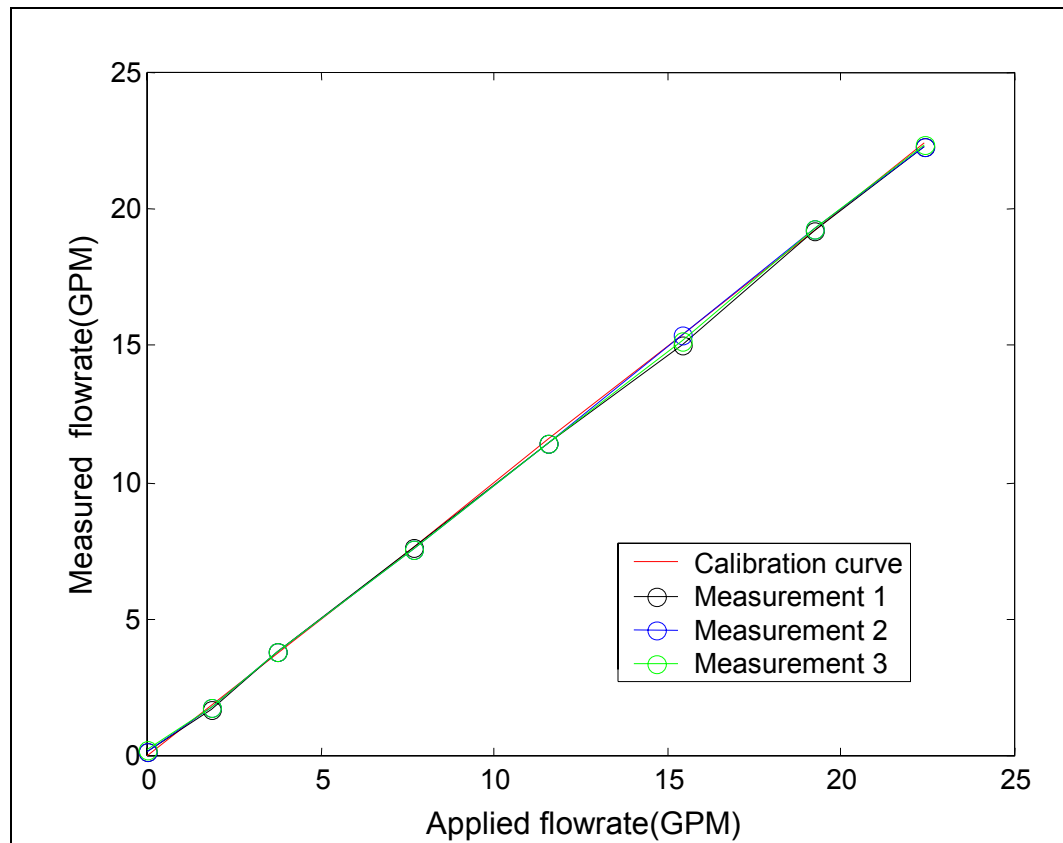

(a) Flow sensor measurement results

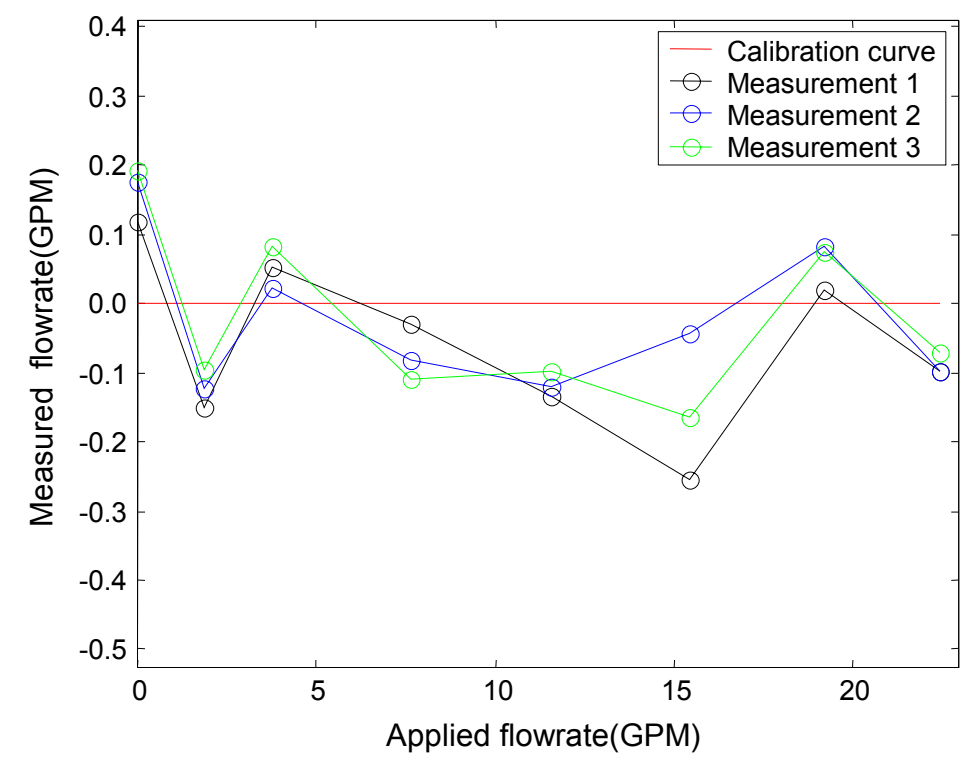

(b) Deviation of flow measurement with respect to calibration data

Figure 4.4. Measurement results from fiber flow sensor in indoor testing loop. 


\subsubsection{Resolution of indoor flow testing}

For the indoor flow sensor system, 600 flow values were acquired in one minute with a sample frequency of 10 counts/second (Figure 4.5). The standard deviation of the flow data was $\sigma=0.00123 \mathrm{GPM}$; therefore, the resolution of the sensor system was estimated to be $2 \sigma=0.0246 \mathrm{GPM}$. The normalized resolution with respect to the dynamic range of the system was $0.11 \%$ of the full scale.

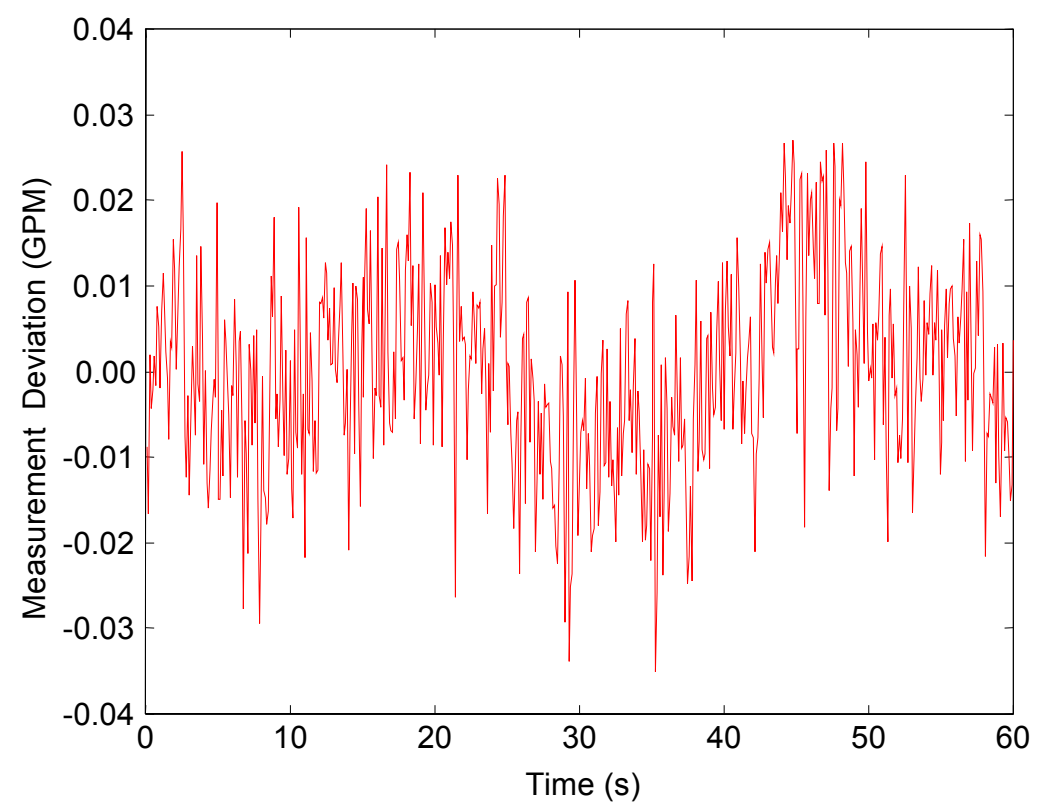

Figure 4.5. Resolution of flow sensor system, indoor flow testing.

\subsubsection{Hysteresis of flow sensor}

The hysteresis characteristics of this sensor were also tested in the indoor flow loop (Figure 4.6a). No obvious hysteresis was observed for the measured range, as shown in the enlarged data in Figure 4.6b. The hysteresis of the full measurement range was measured to be $0.89 \%$ for the indoor flow tests. 


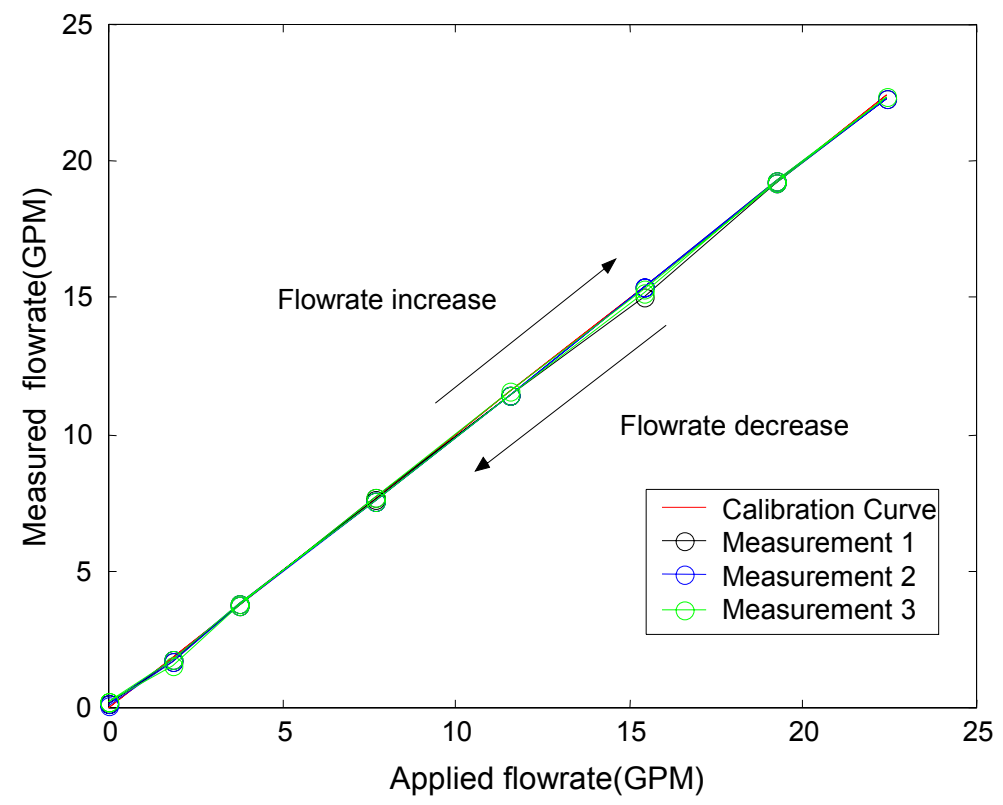

(a) Flow measurement hysteresis test results

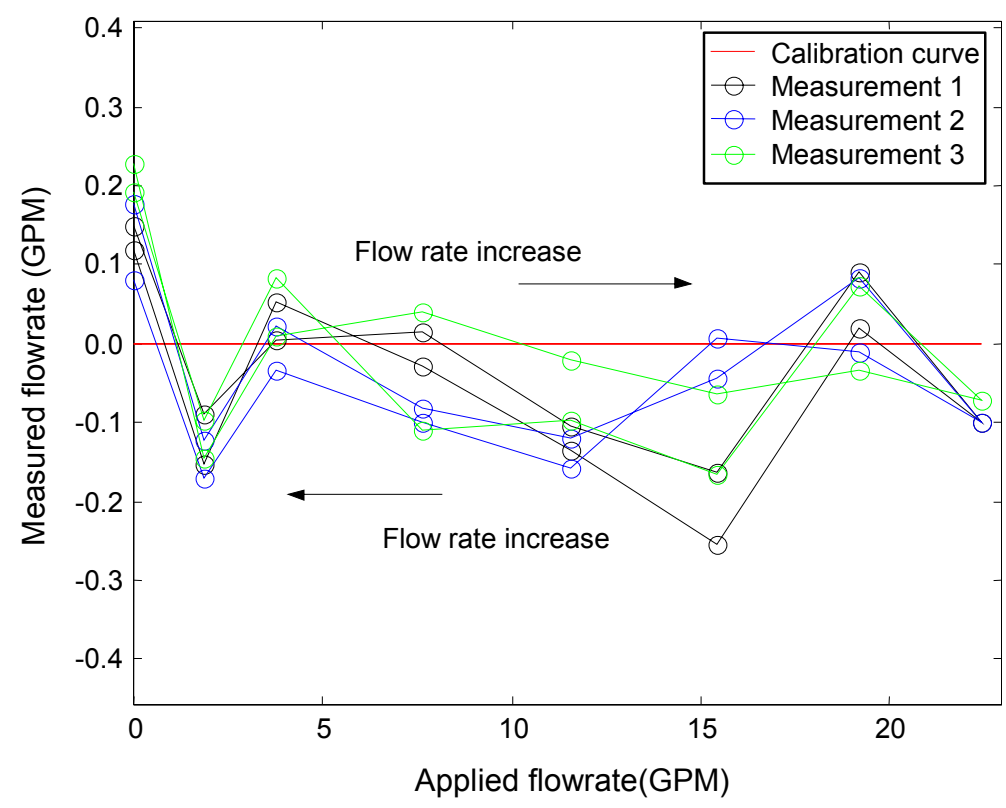

(b) Deviation of the measured flow with respect to the calibration data

Figure 4.6. Flow sensor hysteresis measurement. 


\subsubsection{Stability testing}

To further evaluate the sensor performance in the indoor flow loop, the stability of the designed sensor system was tested as shown in Figure 4.7. With the flow rate maintained at 4GPM for two hours, the output of the fiber optic flow sensing system was within $0.15 \mathrm{GPM}$; the stability of the system was $0.67 \%$ of the full range.

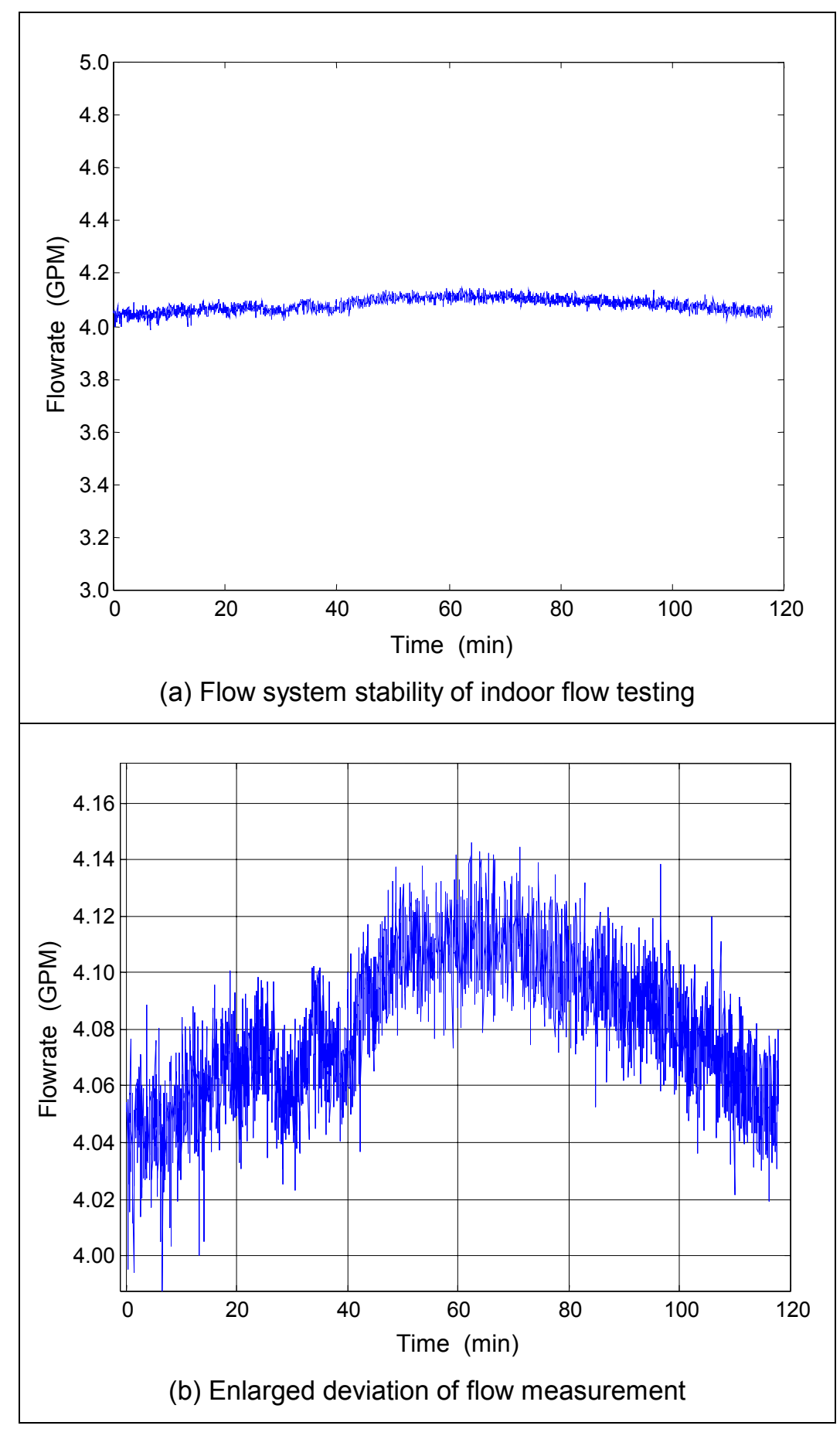

Figure 4.7. Flow system stability test over approximately 2 hours. 


\subsection{Outdoor Flow Loop Testing System and Results}

The outdoor flow testing facility consisted of a simulated oil field flow environment combining pipes from 3 to 6 inches in diameter at various stages (Figure 4.8a). A variable frequency pump (Figure 4.8b)(0 2000GPM) from Waukesha, Inc was used for pumping water from the 3500-gallon tank shown in Figure 4.8c. The Micro Motion vibration flow meter shown in Figure 4.8d was used as the calibration. Many pressure and temperature gauges (Figure 4.8e) from Rosemount Incare were also distributed along the flow pipes to monitor the environmental conditions. The entire facility and gauges were controlled by the central computer in the control room. As shown in Figure 4.9, the fiber optic flow sensor was deployed in the outdoor 3 -inch flow pipes using a 1.5 to 0.75 inch NPT bushing through into a 0.75 inch welding port. The sensor probe was connected to the sensor system optoelectronic unit in the control room through a dual-core multimode fiber cable. The white light interferometer-based flow sensor control system is shown in Figure 4.10a and the flow rate measurement output window in Figure 4.10b. Using the Micro Motion flow meter as a reference, the sensor system was calibrated and tested with the water circulating in the flow loop at different flow rates. After spectrum detection and signal processing, the output flow rate data were digitized, displayed, and recorded.

Due to the complex structure of the outdoor flow testing system, for each testing step a minimum of 20 minutes was required to obtain a relatively stable flow rate. The flow meter and pump were designed for the flow rate range of $0 \sim 2000 \mathrm{GPM}$, with $\pm 0.2 \%$ accuracy of full range, around $\pm 4 \mathrm{GPM}$. So in the relatively low flow rate range of $0 \sim 64 \mathrm{GPM}$, the accuracy of the reference flow meter was diminished. When the pump was set to a certain value, the flow rate fluctuated significantly, affecting the evaluation of the fiber optic sensor system's performance. The flow sensor was calibrated and tested based on the average outputs of the Micro Motion flow meter. The outdoor flow loop testing system experienced pressure fluctuations of around 9 66Psi, and temperature changes of approximately 26 to $37^{\circ} \mathrm{C}$. The flow sensor parameters for outdoor flow testing are listed in Table 4.2.

Table 4.2. Fiber optic flow sensor parameters for outdoor flow testing in Tulsa, OK.

\begin{tabular}{ll}
\hline Fiber type: & Multimode 50/125 \\
Cantilever beam material: & Invar sheet \\
Cantilever beam size: & $0.012 " \times 1.5^{\prime \prime} \times 0.13^{\prime \prime}$ \\
Original air gaps of two sensors: & $9.27 \mu \mathrm{m} ; 9.61 \mu \mathrm{m}$ \\
Original visibility of two sensors: & $52 \% ; 29 \%$ \\
Gauge length of two sensors: & $1.7 \mathrm{~mm} ; 178 \mathrm{~mm}$ \\
Relative temperature coefficients: & $1 ; 1$ \\
Sensor protection: & Flexible Ni-bellows \\
Test conditions: & $84 \sim 86^{\circ} \mathrm{F} ; 1 \sim 5 \mathrm{psi}$ \\
\hline
\end{tabular}




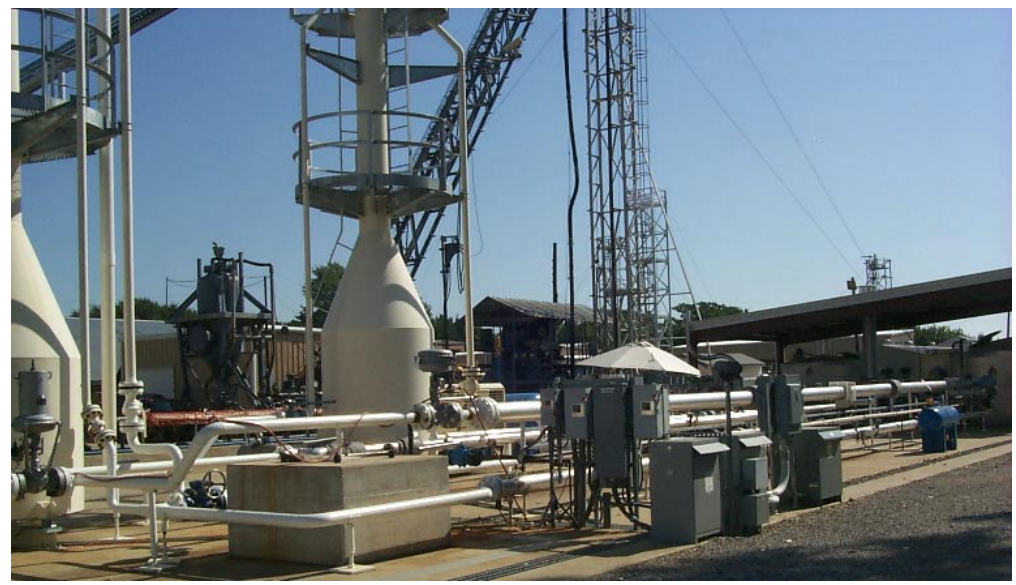

(a) Outdoor flow testing loop

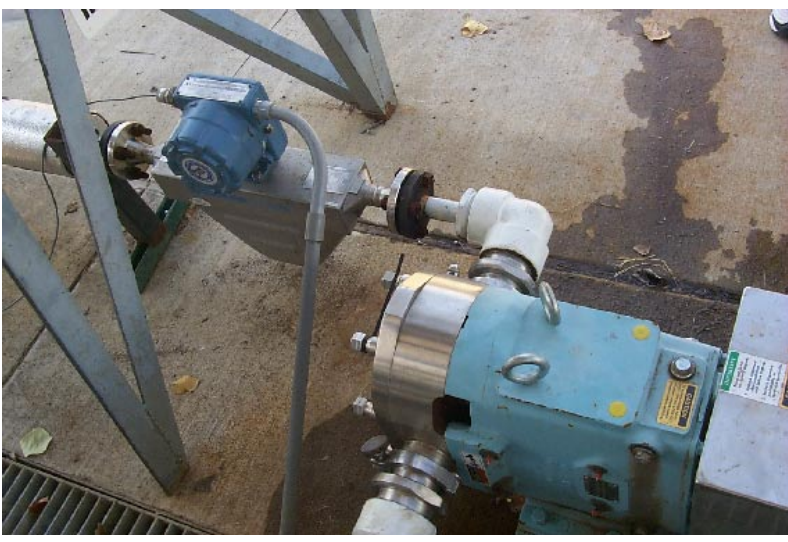

(b) Variable frequency pump (0 2000GPM)

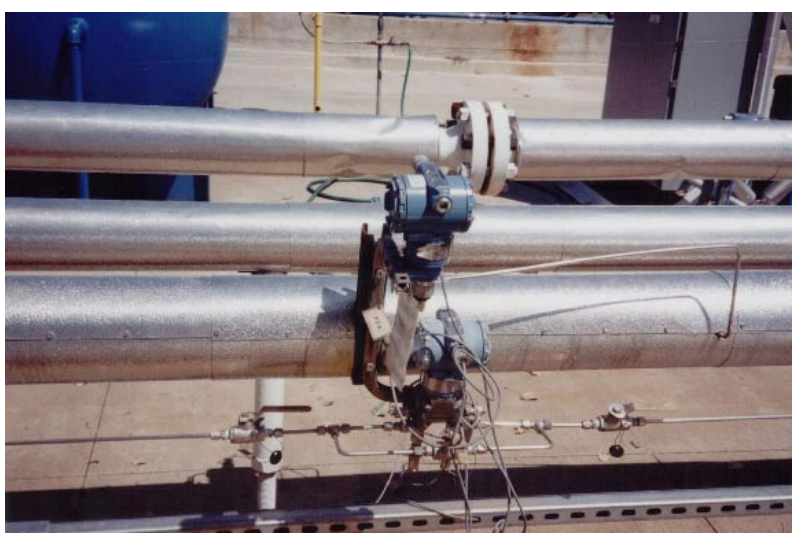

(d) Flow meter in outdoor flow loop

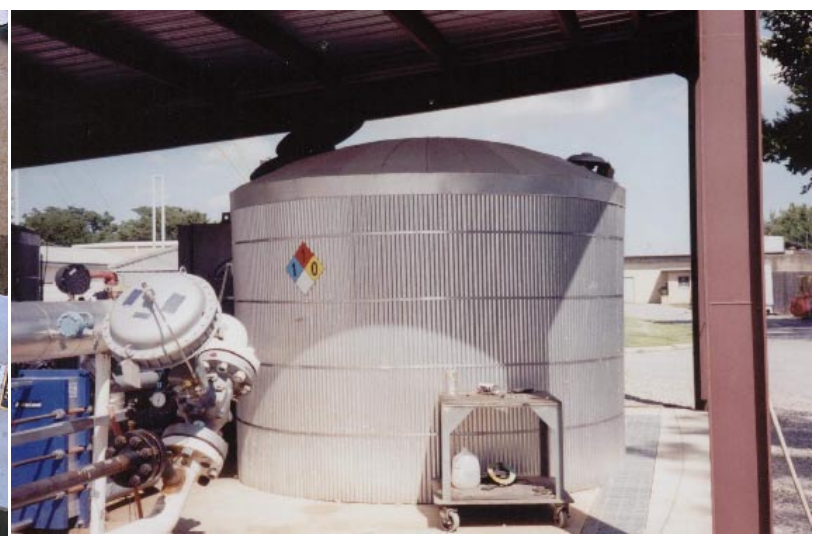

(c) Water tank for outdoor flow testing loop

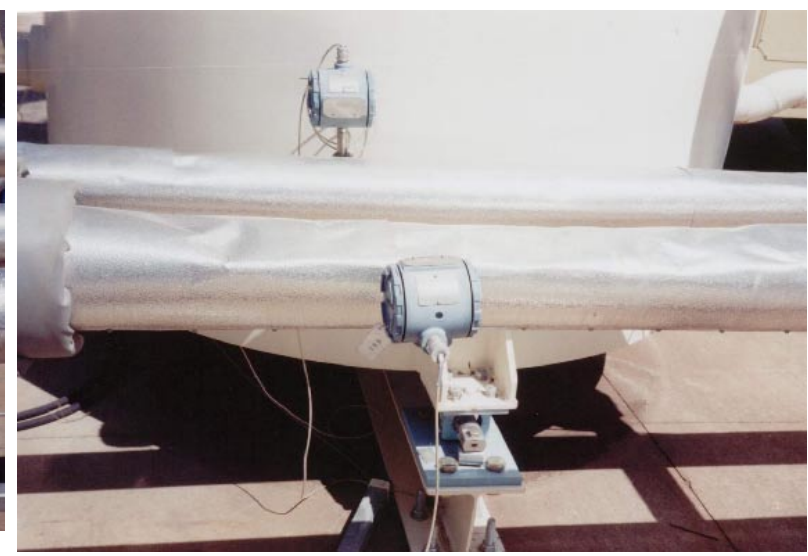

(e) Pressure and temperature gauge in outdoor flow loop

Figure 4.8. Outdoor flow testing facility in Tulsa University, $O K$. 


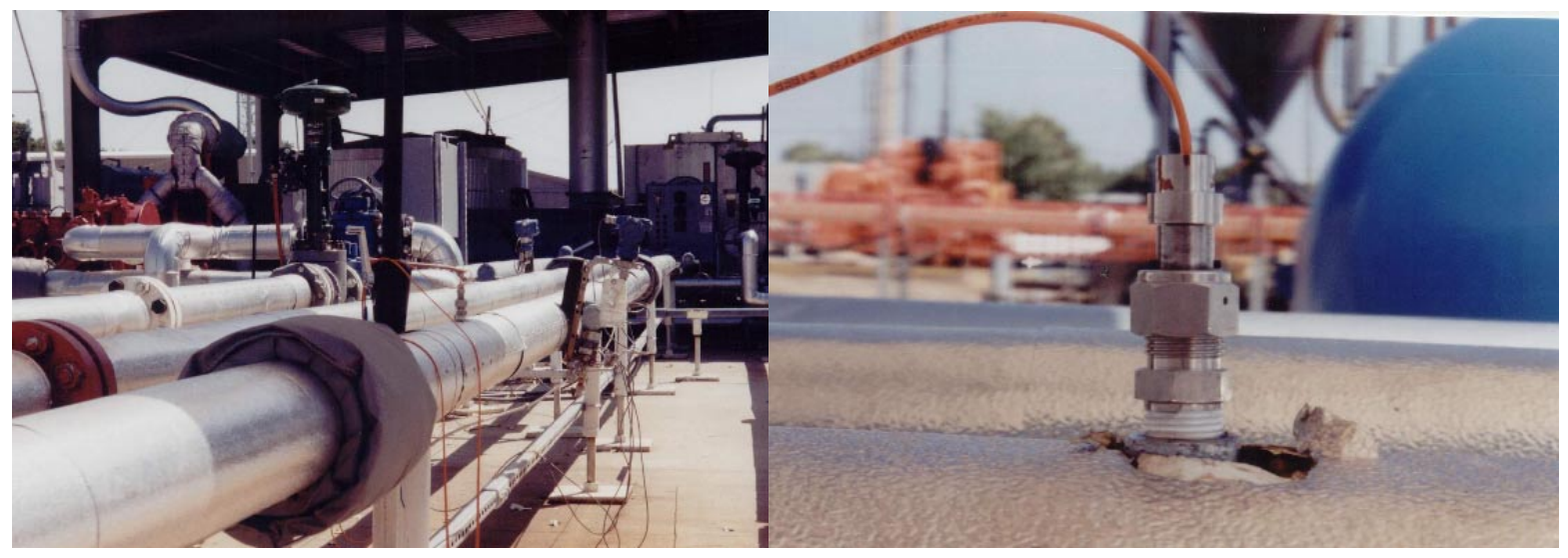

Figure 4.9. Fiber optic flow sensor deployment in outdoor flow loops of Tulsa University, OK.

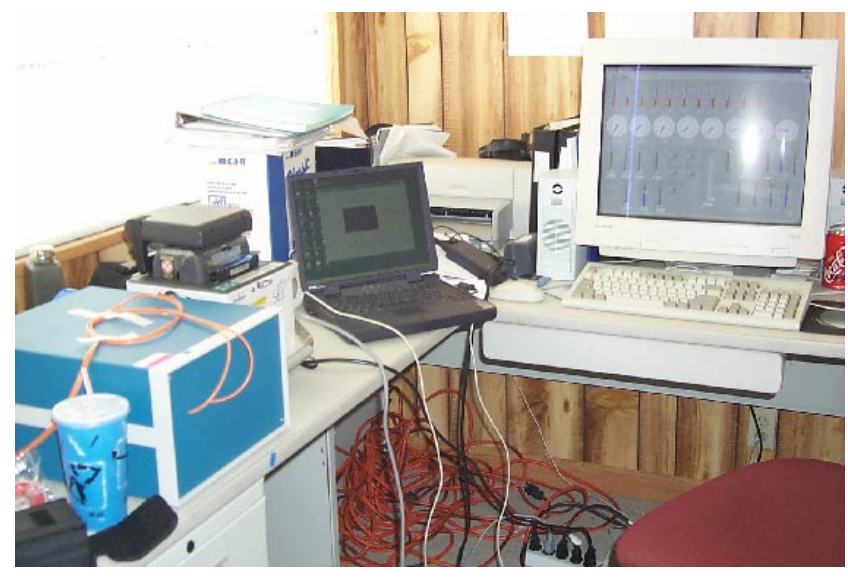

(a) Flow sensor measurement system in the control room of outdoor flow facility

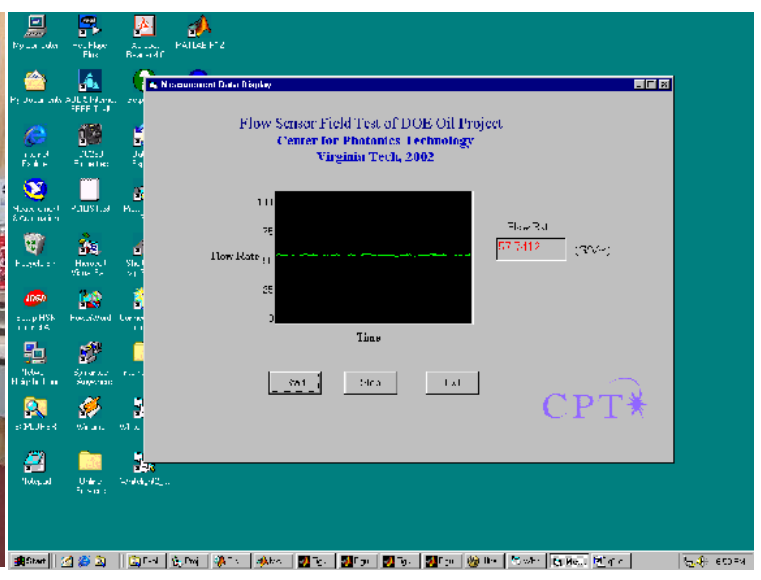

(b) Output window of flow sensor

Figure 4.10. Fiber optic flow sensor control system for outdoor flow testing.

\subsubsection{Calibration}

Due to the difference in testing conditions (including pipe size, sensor deployment position and fluid characteristics) between the outdoor flow loop and lab-scale testing setup, the sensor required recalibration prior to testing in the outdoor system. Figure 4.11a shows the air-gaps of the two sensors and the output of the system with changes in the flow rate during the calibration. The calibration curve was generated as shown in Figure $4.11 \mathrm{~b}$, which provided the compensation coefficients used for the following measurement and evaluation. 


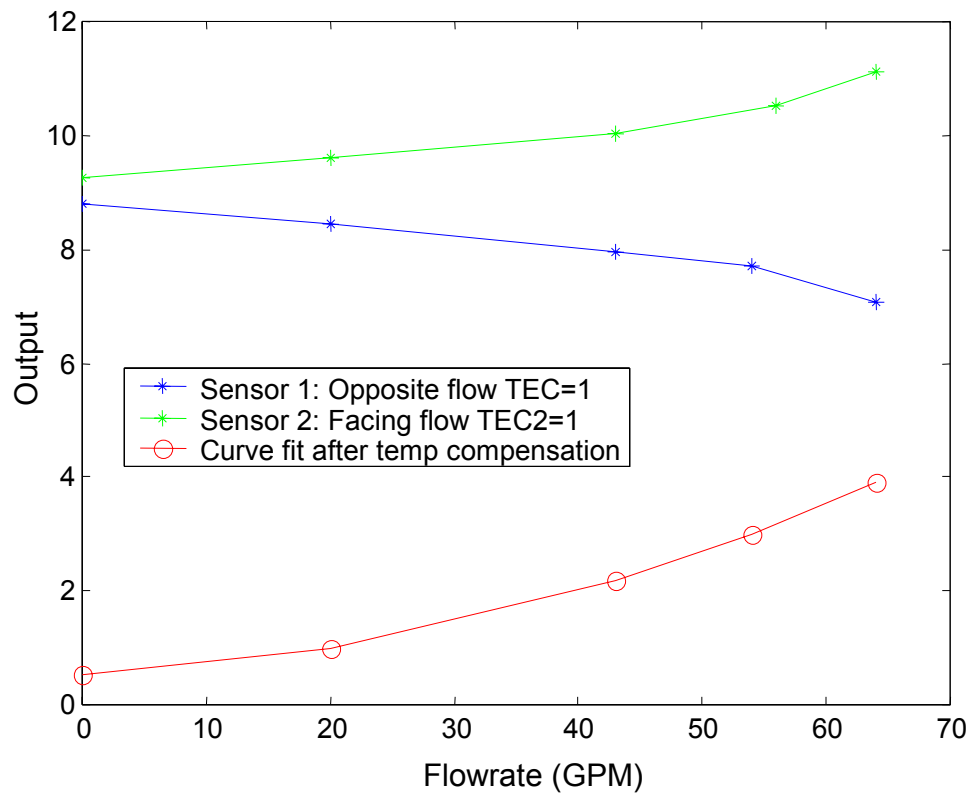

(a) Flow sensor calibration for outdoor flow testing

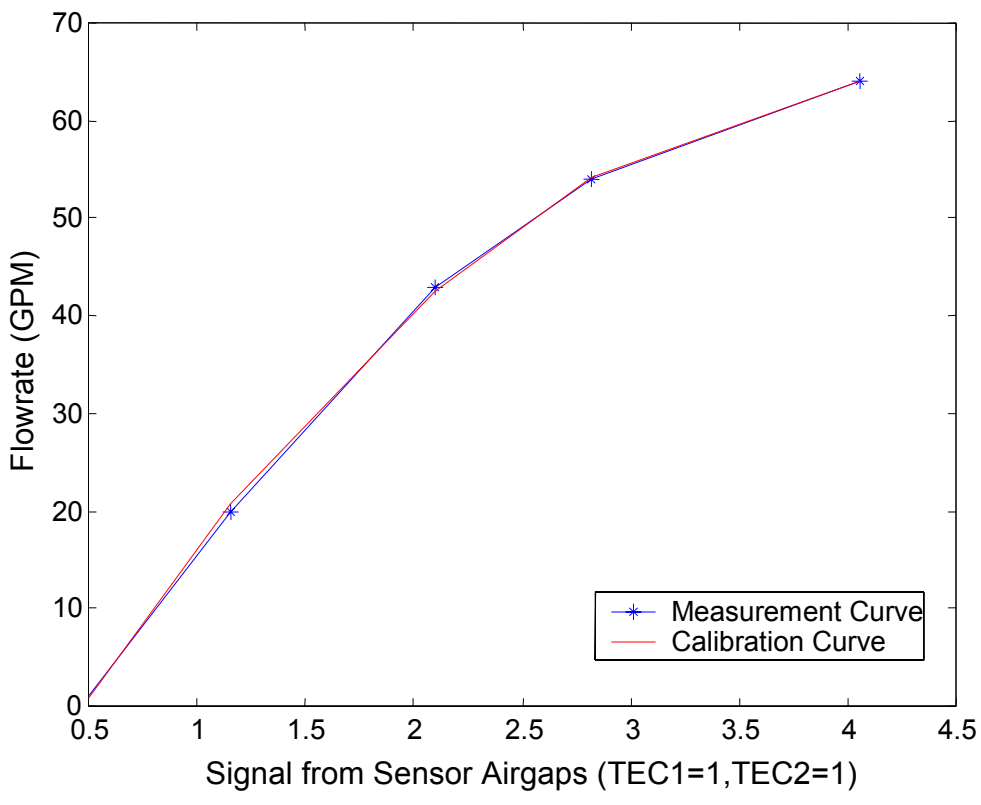

(b) Calibration curve of flow sensor for outdoor flow testing

Figure 4.11. Flow sensor calibration for outdoor flow testing. 


\subsubsection{Stability}

The stability of both Micro Motion flow meter and the fiber flow sensor are shown in Figure 4.12. Maintaining the variable frequency pump at $57 \mathrm{GPM}$ for 70 minutes, the maximum peak-to-peak fluctuation of the reference flow meter was 3.32GPM, which larger than that of the fiber optic flow sensor whose maximum fluctuation is $3.15 \mathrm{GPM}$. Also the standard deviation of the reference flow meter was $0.61 \mathrm{GPM}$, while of the fiber optic flow sensor was 0.42GPM. Relative to the Micro Motion flow meter, the mean deviation of fiber optic flow sensor was $0.9327 \mathrm{GPM}$. Based on this data, we believed that the standard deviation of fiber flow sensor was less than $1 \mathrm{GPM}$, and the stability of this sensor system was better than $1.56 \%$ for $0 \sim 64 \mathrm{GPM}$ full scale.
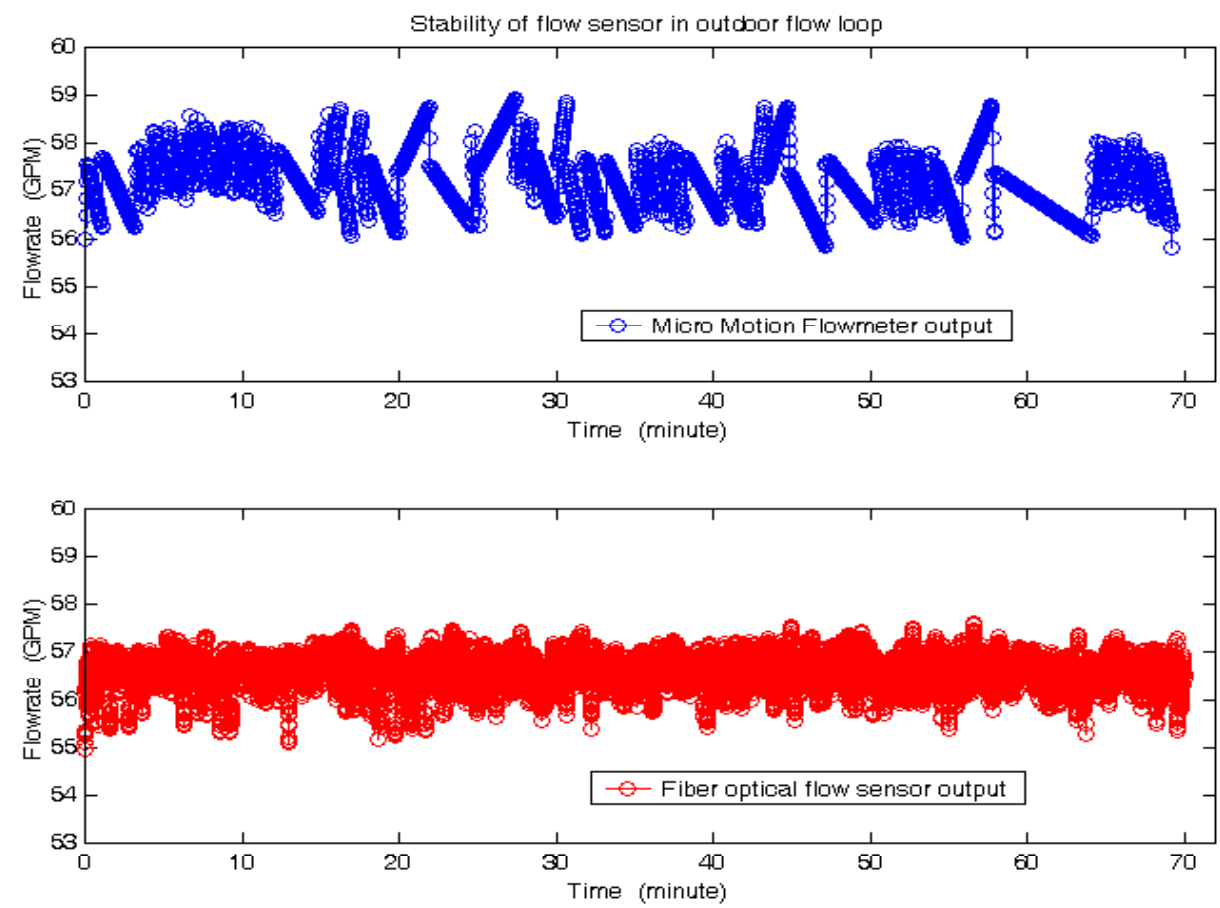

Figure 4.12. Flow sensor stability during outdoor flow testing.

\subsubsection{Resolution}

Using the same definition of resolution as described previously, the resolution of the fiber sensor system in the outdoor testing system is shown in Figure 4.13, and the calculated standard deviation from this data was $\sigma=0.0083 \mathrm{GPM}$. The resolution of the fiber optic sensor system was estimated to be $2 \sigma=0.1664 \mathrm{GPM}$. The normalized resolution for this sensor system was $0.26 \%$ of the full scale. 


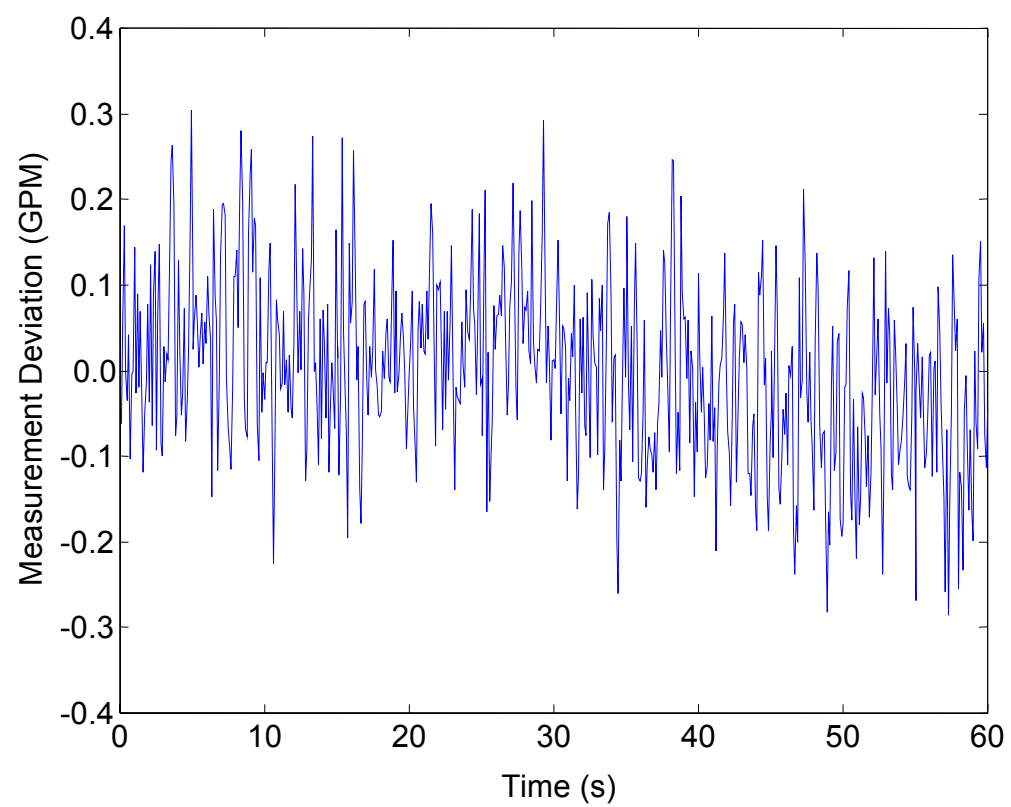

Figure 4.13. Resolution of fiber flow sensor during outdoor flow testing.

\subsubsection{Repeatability}

The flow rate in outdoor flow loop was measured based on above calibration curve and coefficients. Fig 28 shows the measurement results of three repetitions flow rate measurements. Respect to flow meter output, the deviation of these repeatable measurements were enlarged and shown in Fig 28(b), the maximum deviation was $\pm 0.8 \mathrm{GPM}$, which is $\pm 1.25 \%$ of full scale.

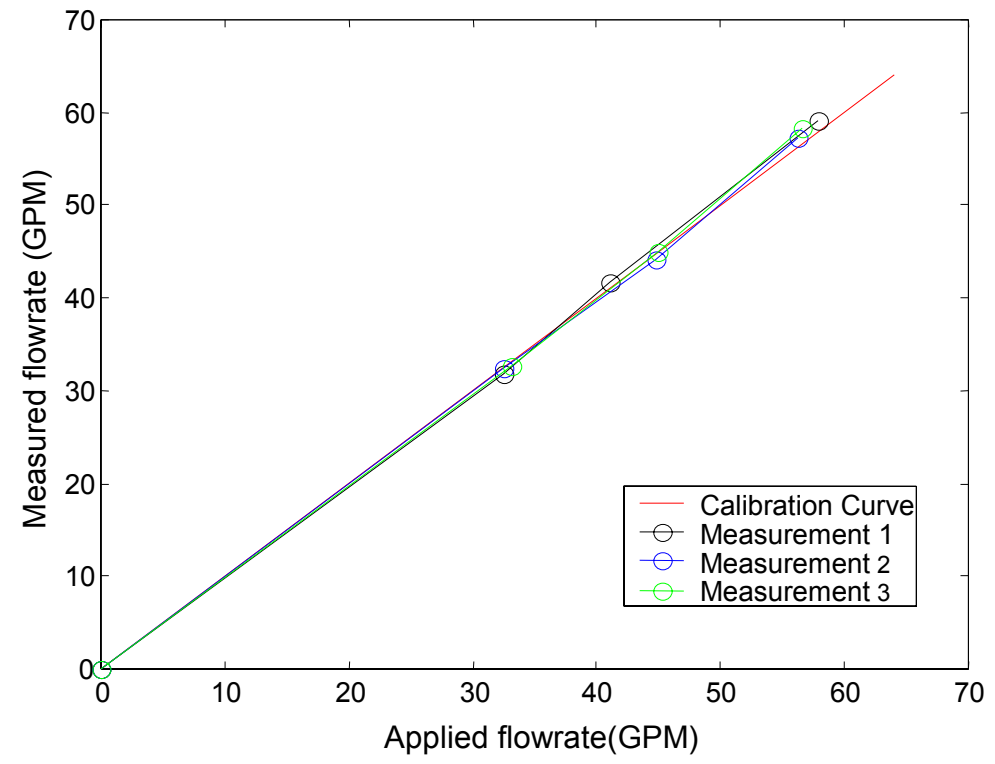

(a) Measurement results in outdoor flow testing 


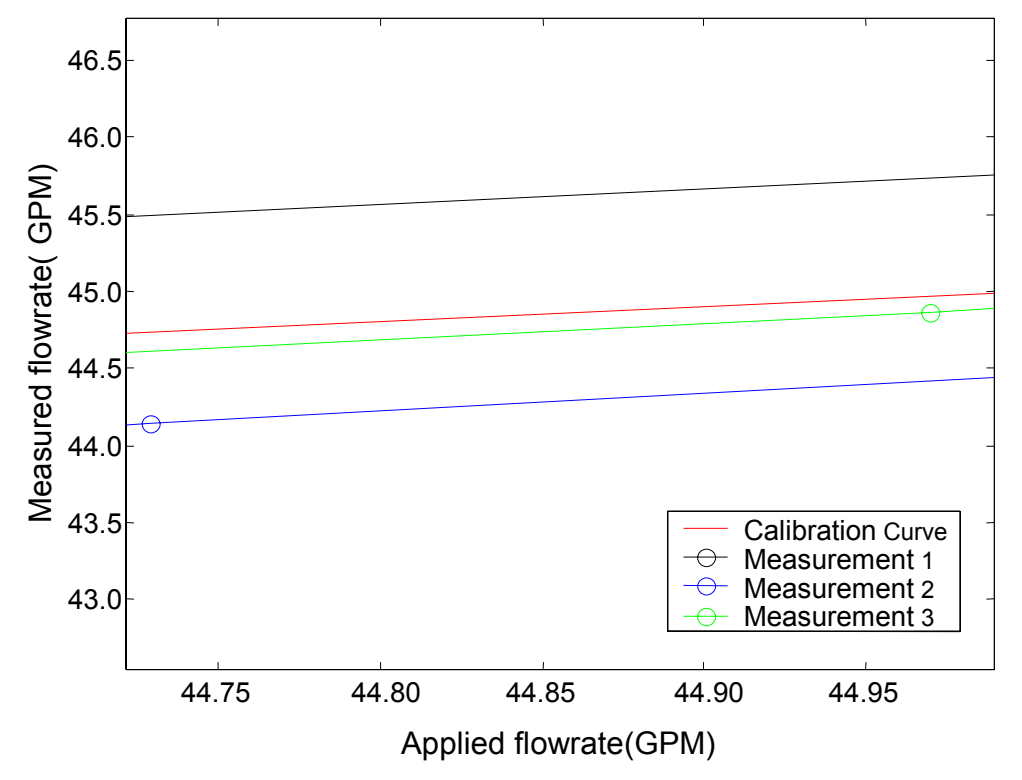

(b) Enlarged deviation in measurement results in outdoor flow loop

Figure 4.14. Measurement results and repeatability testing in outdoor flow testing.

\subsubsection{Hysteresis}

As shown in Figure 4.15, the hysteresis of the flow measurement was evaluated as $2.1 \%$ of full range during outdoor flow loop testing. Further improvement in sensor performance may be achieved by improving the sensor materials and fabrication techniques, the accuracy of the reference flow rate as well as the test system stability with respect to laminar flow generation. 


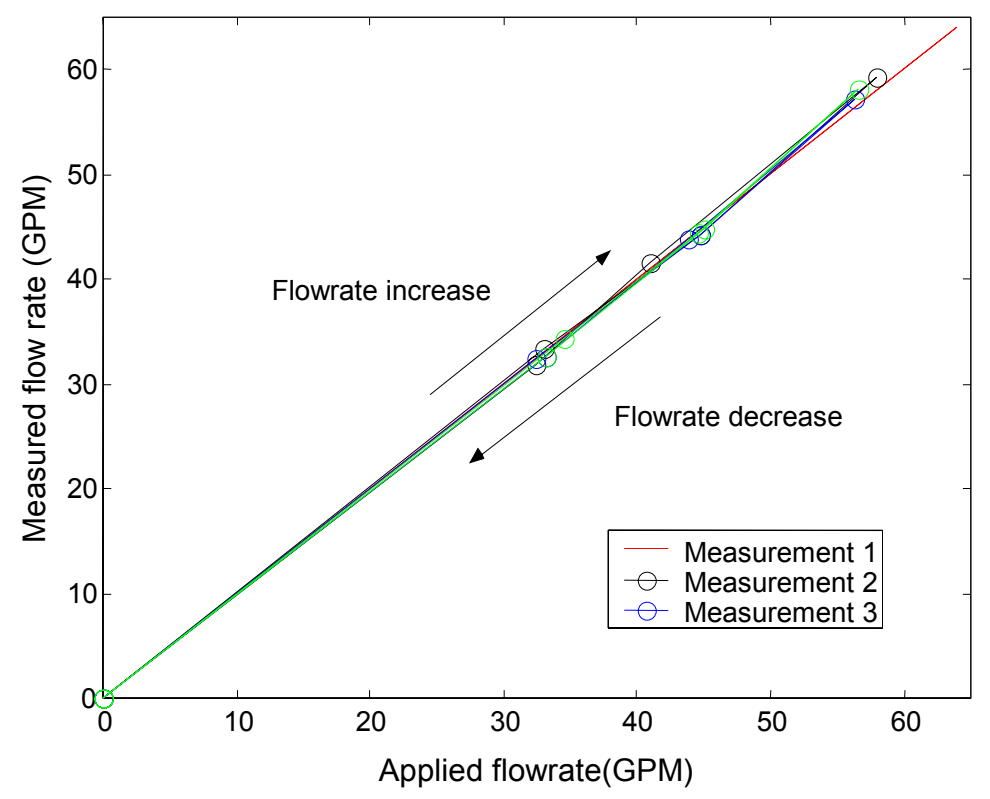

(a) Hysteresis testing in outdoor flow testing

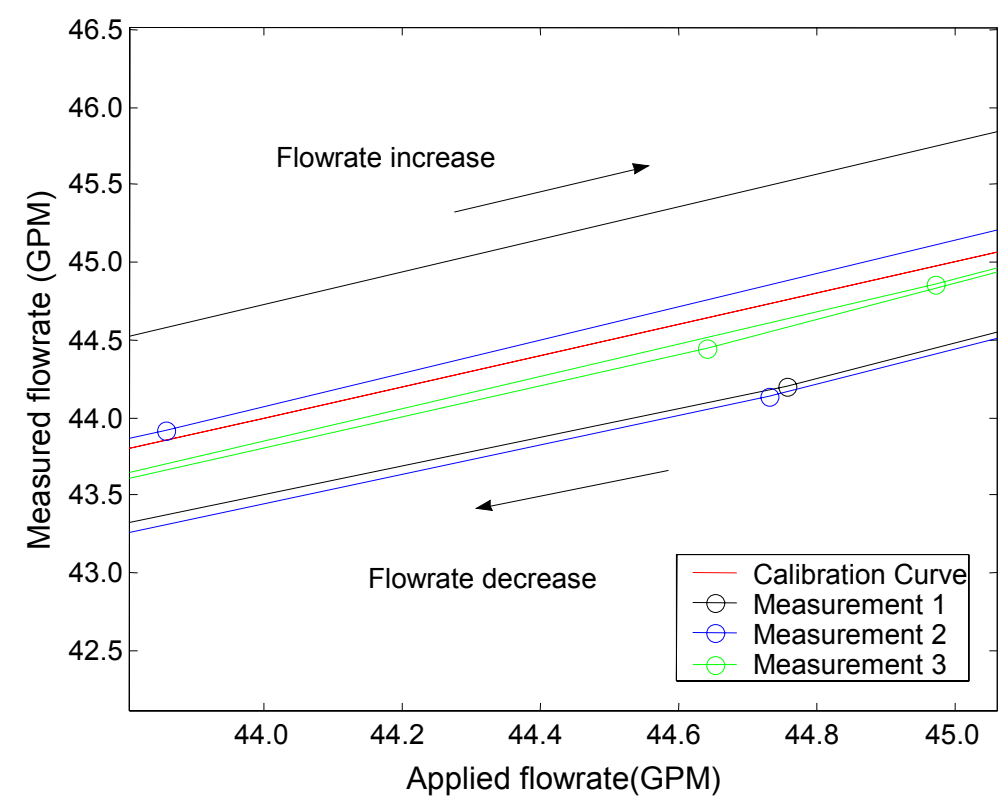

(b) Enlarged deviation during hysteresis testing in outdoor flow loop

Figure 4.15. Hysteresis testing in outdoor flow testing. 


\subsection{Temperature and Pressure Sensor Field Testing}

To evaluate the performance of the fiber optic sensor system in the oil site, a multimode white light sensor system was tested at the oil site of Chevron/Texaco Company (Coalinga CA). The sensors were deployed using a hydraulic optic fiber deployment system developed by the Chevron/Texaco Company. The position of the sensor head in the oil well can be monitored by OTDR. After deployment, a pump was used to increase the pressure inside the testing pipe to about $3000 \mathrm{Psi}$, and then all valves were shut off to maintain a constant pressure inside the pipe. As discussed in the previous report, the sensor system was accessed remotely to control the host computer and obtain the measurement data. Data was saved to the hard disk of the host computer once per minute. The software generated a new filename at time 00:00:00 each day so that one file contained one day's measurement data. Figure 5.1 and Figure 5.2 show the testing results through the end of this reporting period.

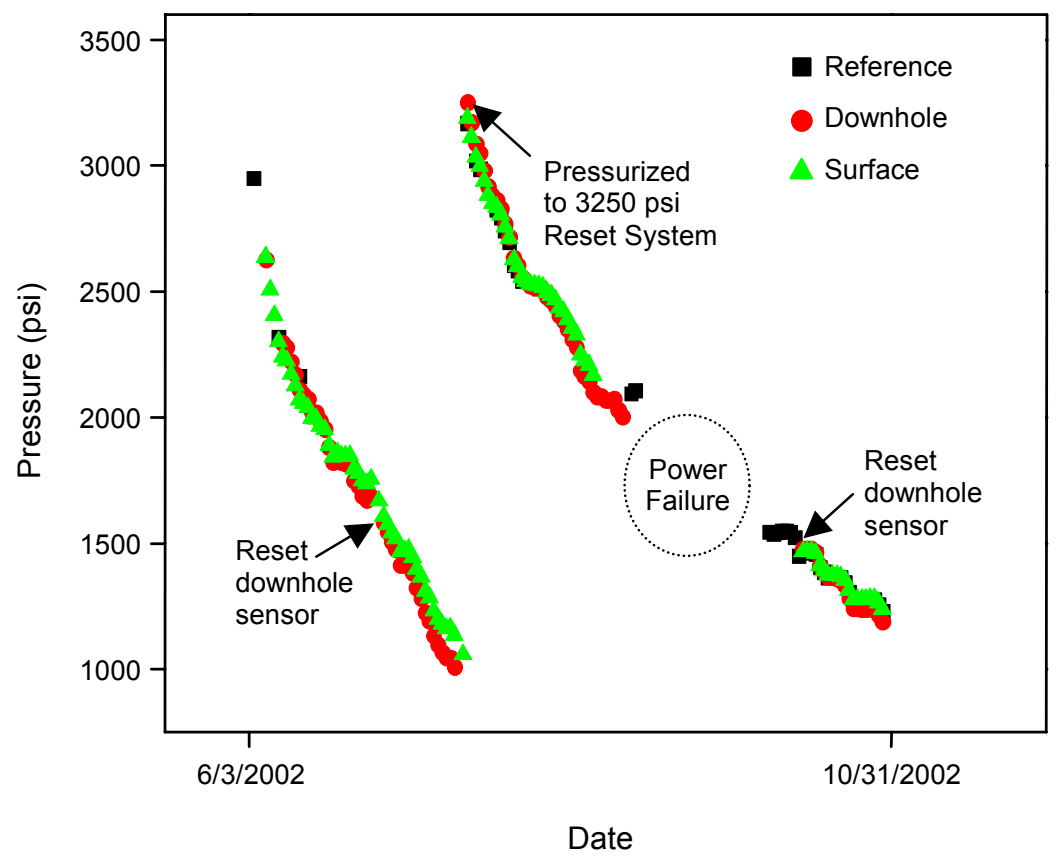

Figure 5.1. Pressure sensor output from 6/3/2002 - 10/31/2002. 


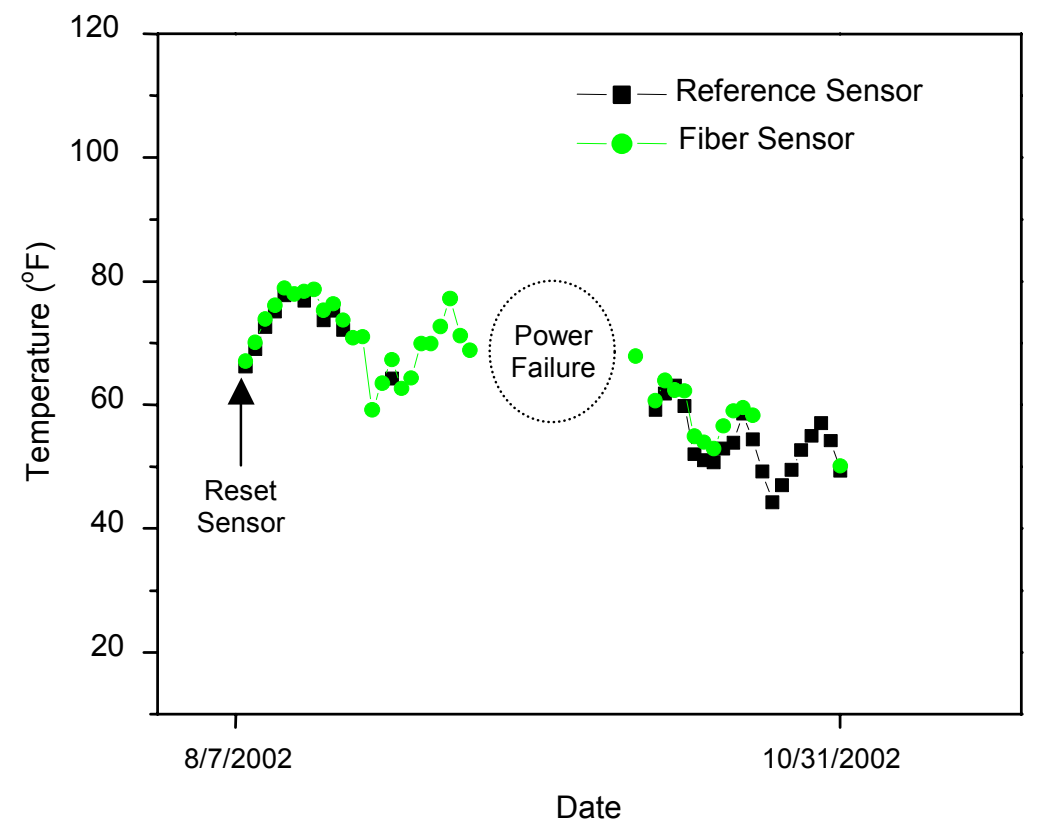

Figure 5.2. Temperature sensor output from 8/7/2002 - 10/31/2002. 


\subsection{Conclusions}

To fulfill the objective of providing robust and reliable fiber optic flow sensors capable of operating in harsh environments, a novel fiber optic flow sensor system has been manufactured at Virginia Tech's Center for Photonics Technology (CPT). By combining various commercial and reported flow meters or sensors with CPT's strong fiber optic sensor background, a self-compensating fiber optic flow sensor based on a cantilever beam and interferometer was designed that can be easily inserted into the flow pipe. Theoretical analysis and simulations have been performed to ensure of the correctness of the design. The sensor fabrication and packaging techniques have been investigated and improved. Prototype flow sensors have been fabricated based on the fabrication experience of hundreds of test sensors. A lab-scale flow testing system has been constructed and used for sensor evaluation. Field-testing has been performed in both the indoor and outdoor flow testing facility at The University of Tulsa, OK. Comprehensive testing was performed to systematically evaluate the performance of this fiber optic flow sensor system in both lab and field flow environments. The major test results are summarized in Table 6.1, which give us confidence that the developed fiber optic flow sensor system provides a reliable tool for flow measurements in harsh environments. This research clearly demonstrates the feasibility of the developed fiber optic flow sensor system for real-time flow rate measurements in the fluid filled pipes of oil field.

Table 6.1. Summary of the performance evaluation results for SCIIB flow sensor system.

\begin{tabular}{lcccc}
\hline & Repeatability & Hysteresis & Resolution & Stability \\
\hline Lab-scale testing & $\pm 1.62 \%$ & $1.35 \%$ & $0.11 \%$ & $<1.89 \%$ \\
& & & & $(\sim 12 \mathrm{hrs})$ \\
Indoor flow loop testing & $\pm 1.15 \%$ & $0.89 \%$ & $<0.11 \%$ & $0.67 \%$ \\
& & & & $(\sim 2 \mathrm{hrs})$ \\
Outdoor flow loop testing & $\pm 1.25 \%$ & $2.1 \%$ & $0.26 \%$ & $<1.56 \%$ \\
& & & $(\sim 70 \mathrm{~min})$ \\
\hline
\end{tabular}

Based on the sensor development to date, some future work is required to further enhance the robustness and reliability of the flow sensor systems for conducting accurate flow measurements in practical oil field applications, including improving the sensor materials and fabrication techniques, improving the accuracy of the reference flow rate as well as the test system stability with respect to laminar flow generation, and also extending this flow sensor to multiphase flow measurement. 


\subsection{References}

1. D. B. Marghitu, "Materials Engineering Handbook," in Materials Engineering Handbook: Academic Press, pp. 131-135 (2001).

2. A. Wang, H. Xiao, J. Wang, Z. Wang, W. Zhao, and R. G. May, "Self-Calibrated Interferometric/Intensity-Based Optical Fiber Sensors," J. Lightwave Tech. 19 (10), pp. 1495-1501 (2001).

3. S. Webster, R. McBride, J. S. Barton, and J. D. C. Jones, "Air flow measurement by Vortex shedding from multimode and monomode optic fibers," Meas. Sci. Technol 3, pp. 210-216 (Mar 1992).

4. B. Qi, G. R. Pickrell, J. Xu, P. Zhang, Y. Duan, W. Peng, Z. Huang, R. G. May, and A. Wang, "High-resolution white light interferometer and its application," submitted to Applied Optics. 


\section{Bibliography}

1. G. S. Brady, H. R. Clanser, and J. A. Vaccari, in Materials Handbook, pp. 518 (1997).

2. N. P. Cheremisinoff, Applied fluid flow measurement - fundamentals and technology. New York: M. Dekker (1979).

3. N. P. Cheremisinoff and P. N. Cheremisinoff, Flow measurement for engineers and scientists. New York: M. Dekker (1988).

4. J. Dakin and B. Culshaw, Optical Fiber Sensors: Principles and Components. Boston: Artech House (1988).

5. J. X. Fang, H. F. Taylor, and H. S. Choi, "Fiber-optic Fabry-perot flow sensor," Microwave and Optical Technology Letters 18, pp. 209-211 (Mar 1998).

6. Y. Imai and K. Tanaka, "Direct velocity sensing of flowing distribution based on lowcoherence interferometry," J. Opt.Soc.Am.A 16, pp. 2007-2011 (Aug 1999).

7. S. W. James, R. A. Lockey, D. Egan, and R. P. Tatam, "Fiber optic reference beam laser Doppler velocimetry," Flow. Meas.Instrum. B2B, pp. $89-92$ (1995).

8. F. E. Jones, Techniques and topics in flow measurement: CRC Press (1995).

9. P. A. Leilabady, J. D. C. Jones, and D. A. Jackson, "Monomode fiber optic interferometric techniques in flow velocity measurement," Opt.Acta 132, pp. 233-240 (Feb 1985).

10. P. A. Leilabady, J. D. C. Jones, A. D. Kersey, M. Corke, and D. A. Jackson, "Monomode fiber optic vortex shedding flowmeter," Electronics Letters 20, pp. 664-665 (Aug 1984).

11. J. H. Lyle and C. W. Pitt, "Vortex shedding fluid flowmeter using optic fiber sensor," Electronics Letters 17, pp. 244-245 (March 1981).

12. D. B. Marghitu, "Materials Engineering Handbook," in Materials Engineering Handbook: Academic Press, pp. 131-135 (2001).

13. R. W. Miller, in Flow measurement engineering handbook: McGraw-Hill, pp. 14-16 (1989).

14. B. Qi, G. R. Pickrell, J. Xu, P. Zhang, Y. Duan, W. Peng, Z. Huang, R. G. May, and A. Wang, "High-resolution white light interferometer and its application," submitted to Applied Optics.

15. E. Udd, Fiber Optic Sensors-An Introduction for Engineers and Scientists. New York: Wiley (1991).

16. A. Wang, M. S. Miller, A. J. Plante, M. F. Gunther, K. A. Murphy, and R. O. Claus, "Split-spectrum intensity-based optical fiber sensors for measurement of microdisplacement, strain and pressure," Applied Optics 35 (15), pp. 2595-2601 (1996).

17. A. Wang, H. Xiao, J. Wang, Z. Wang, W. Zhao, and R. G. May, "Self-Calibrated Interferometric/Intensity-Based Optical Fiber Sensors," J. Lightwave Tech. 19 (10), pp. 1495-1501 (2001).

18. W.-C. Wang, S. Yee, and P. G. Reinhall, "Fluid Viscosity and mass flow sensor using forward light scatterin," Proc. SPIE 2574, pp. 146-151 (1995).

19. W. M. Wang, W. J. O. Boyle, K. T. V. Grattan, and A. W. Palmer, "Fiber-optic Doppler velocimeter that incorporates active optical feedback from a diode laser," Optics Letters 17 (11), pp. 819-821 (Jun 1992).

20. M. J. Weber, in Optical Materials: CRC Press, pp. $72-73$ (1995). 
21. S. Webster, R. McBride, J. S. Barton, and J. D. C. Jones, "Air flow measurement by Vortex shedding from Multimode and monomode optic fibers," Meas. Sci. Technol 3, pp. 210-216 (Mar 1992) 


\section{List of Acronyms and Abbreviations}

A/D, analog to digital
APP, Advanced Pressure Products, Inc.
CCD, charge couple device
CPT, Center for Photonics Technology
CTE, coefficient of thermal expansion
EFPI, extrinsic Fabry-Perot interferometer
EMI, electromagnetic interference
FWHM, full width half maximum
GPM, gallons per minute
GRIN, graded index
LED, light emitting diode
MMF, multimode fiber
PC, personal computer
PZT, lead zirconium titanate
SCIIB, self-calibrated interferometric/intensity-based
SLED, superluminescent light emitting diode
SMF, single mode fiber
SNR, signal to noise ratio
VTPL, Virginia Tech Photonics Laboratory (now Center for Photonics Technology) 